\title{
EMPLOYMENT PRECARIOUSNESS AND MENTAL HEALTH - UNDERSTANDING A COMPLEX REALITY: A SYSTEMATIC REVIEW
}

\author{
MIREIA UTZET ${ }^{1,2}$, ERIKA VALER0 $^{1,2}$, ISABEL MOSQUERA $^{1,2}$, and UNAI MARTIN ${ }^{1,2}$ \\ ${ }^{1}$ University of the Basque Country UPV/EHU, Leioa, Spain \\ Department of Sociology 2 \\ ${ }^{2}$ Social Determinants of Health and Demographic Change - Opik, Leioa, Spain
}

\begin{abstract}
Precarious employment has expanded during the last decades, but there is no full consensus on its definition, and its impact on mental health is not completely understood. The relevance of several micro- and macro-level variables in the association between precarious employment and mental health has not been fully addressed. This review has 2 aims: to identify scientific evidence on the relationship between various dimensions of precarious employment and mental health, and to synthesize the inclusion of a gender-sensitive perspective, context variables, workers' household variables, and the discussion of causal mechanisms underlying the association. The literature was searched in PubMed, EMBASE, Web of Science and PsycINFO including articles dated 2010-May 2018. A minimum of 2 independent reviewers assessed each article regarding quality and eligibility criteria. The search retrieved 1522 papers, of which 54 (corresponding to 53 studies) met the inclusion criteria. Most of the studies analyzing job insecurity, temporariness and multidimensional approaches reported a significant association. Nevertheless, results for working time arrangements and downsizing are inconclusive. Around half of the studies included sex-stratified analyses and formulated contradictory conclusions. Overall, 7 studies considered workers' household situation and only 3 delivered significant results, and 16 described some of the potential pathways. There is evidence of an association between various precarious employment approaches and mental health problems. Further research (preferably longitudinal) should aim to discuss theoretical models explaining the pathways between precarious employment and mental health, including a gender-sensitive perspective, and integrating several levels of individual and contextual variables. Int J Occup Med Environ Health. 2020;33(5):569-98
\end{abstract}

Key words:

mental health, review, gender, Europe, pathway, precarious employment

\section{INTRODUCTION}

In modern societies, employment (or the lack of it) is a social determinant of health and, as such, can be a source of social and psychological wellbeing or of alienation and health-related problems [1]. After 30 "golden" post-war years of western capitalist societies, characterized by constant economic growth and a generalized improvement in working conditions (mainly for men), the socioeconomic and political changes that started at the end of the 1970s resulted in the collapse of the normative model of employment. Standard employment with a permanent, full-time contract, a regular and "sufficient" salary, and employment rights shrank while precarious and flexible forms of employment dramatically increased. In this new context, insecurity has become a feature of both the labor market [2] and the professional and social lives of work-

Received: October 1, 2019. Accepted: June 4, 2020.

Corresponding author: Mireia Utzet, University of the Basque Country UPV/EHU, Department of Sociology 2, Campus de Leioa s/n, 48940 Leioa, Spain (e-mail: mireia.utzet@ehu.eus). 
ers, especially in the case of young people, women and immigrants, among whom there is a growing perception that job insecurity is unavoidable [3].

One of the main challenges for research in this field is the lack of a clear definition of precarious employment, beyond it being a social determinant of health [4]. Most studies on precarious employment have been based on a unidimensional approach, and considered precarious employment mainly as job insecurity [5] or temporary work [2]. Although insecurity and temporariness are 2 key dimensions, they represent only part of the concept of precariousness [6]. In order to comprehend the complexity of this concept, there is a need to adopt a multidimensional perspective [7]. In recent years, various multivariate approaches have been developed, such as the proposal by Rodgers [8], the Employment Strain Model [9], and the Employment Precariousness Scale (EPRES) [10]. The common denominator of these definitions is that precarious employment implies a lack of security in some aspect of employment and working conditions, vulnerability, low salaries, and few opportunities for training and professional development.

The impact of temporary employment on health has been identified through a higher risk of work-related injury, an increase in mental health problems and presenteeism, with potential long-term negative effects [11]. On the other hand, perceived job insecurity has been associated with poor mental health [5], and a deterioration in occupational health and safety [12]. Research based on multidimensional approaches to precarious employment and its health consequences is still scarce, although there is a clear progress in this matter [13]. During the last decade, several studies have found evidence that precarious employment has an impact on physical, and particularly mental, health $[14,15]$. Two theoretical reviews published in 2007 [6] and 2014 [4] summarized different models, concepts and findings on precarious employment and its mental health consequences, but a systematic review with quality filters is relevant and still lacking.
A shortcoming in this topic is the scarcity of theoretical frameworks showing the causal mechanisms between different types of precarious employment and health [13]. The main causal pathways proposed in the literature are the financial threat and a potential loss of latent functions of employment when working in precarious conditions [16], the association of precarious employment with higher exposition to hazardous working conditions [17], and the workers' uncertainty and lack of control over their work situation [4]. On top of that, models should include micro- and macro-level factors which individuals are embedded in, and pathways between them, as well as precarious employment and mental health outcomes. Starting at the micro level, studies on the differential impact of precarious employment according to gender, social class, and migration status are needed. Due to gender-related historical factors and gender division in the labor market and housework [18], a gender-sensitive perspective should be included. In addition, workers' social support and household situation may mediate the impact of precarious employment on health $[9,19]$, and little is known about it. Moreover, the macro-level structures of the welfare state and labor market policies are important determinants of the extent of precarious employment and its health consequences [20]. However, the knowledge on the interaction between macro-level structures and individual factors in the association of precarious employment and health is still limited. In this sense, the structural crisis that started in 2008 should be considered. The austerity and labor market reforms implemented in Europe posed threats to both working conditions and workers' health. They led to a deterioration of employment and working conditions, and an increase in the rates of unemployment and precarious employment across Europe [21], with strong effects on health, especially of those already vulnerable [22].

In this complex context, this review aims to update the existing evidence on the relationship between precarious employment (using both unidimensional and multidimen- 
sional approaches) and mental health, to assess which indicators were most widely used, and whether associations with mental health had been previously found. The inclusion of the 4 key aspects in the association was also examined. These aspects were as follows: the incorporation of a gender-sensitive perspective; the discussion of hypothetical causal mechanisms underlying the association; the integration of social, political and economic context variables (specifically, the welfare state policies and the crisis impact); and finally, the inclusion of workers' social support and household variables. Finally, attempts were made to identify gaps in this area of knowledge, pointing out to future lines of research.

\section{METHODS}

A systematic review of the scientific literature was conducted to identify studies on the association between precarious employment and mental health published in 2010-May 2018, based on data concerning the European Union, Norway, Island, Liechtenstein and Switzerland. The start date was chosen because the economic recession peak was reached in 2009, and it was felt that the effects of the crisis and the austerity measures on the labor markets and working conditions would have already begun to be noticed in Europe. The systematic review was conducted according to the systematic literature review guidelines of the Centre for Reviews and Dissemination [23]. Overall, 4 databases were systematically searched: PubMed, EMBASE, Web of Science and PsycINFO.

The definition of the search terms related to precarious employment was developed in 2 steps. Firstly, based on the proposals by Rodgers [8], Amable [7], and Van Aerden [24], 9 dimensions of precarious employment were defined: disempowerment and collective bargaining, downsizing and major organizational restructuring, employee involvement, income wages, job insecurity (fears related to the loss of a current job, finding a job, and changes in working conditions), temporariness, vulnerability, work- ers' rights and working time arrangements. In addition to these individual dimensions, multidimensional approaches to precarious employment were also considered. Secondly, search terms for each of these dimensions were defined, as set out in Table 1. Those search terms included $\mathrm{MeSH}$ and free-text terms related to precarious employment, mental health and European countries.

\section{Selection criteria}

Studies were selected based on the following inclusion criteria:

- observational design;

- active population (aged 18-65 years);

- exposure to precarious employment (defined as single exposures or multidimensional approaches as outlined in the previous paragraph);

- outcomes, including mental health outcomes (excluding suicide and sleeping problems);

- data from European countries (Norway, Island, Liechtenstein and Switzerland, as well as European Union member states, including the UK);

- written in English, Spanish or French;

- published in 2010-May 2018;

- inclusion of statistical results (relative risk, odds ratio, hazard ratio, etc.).

Studies that focused on precarious employment, but did not include an assessment of its association with mental health, were excluded.

\section{Selection process}

The study selection process was also carried out in 2 steps. First, references were selected based on reading their title and abstract. Then, the selection criteria were applied to the full text. All papers were screened by at least 2 reviewers. In the case of disagreements, the decision was discussed until a consensus was reached. Finally, manual search of articles was done, and references cited in the previous reviews in the field were screened. 
Table 1. Search terms used in the systematic review, including MeSH and free-text terms related to precarious employment, mental health and European countries, in the systematic review on employment precariousness and mental health (2010-2018)

\begin{tabular}{|c|c|}
\hline Variable & Search terms \\
\hline \multicolumn{2}{|l|}{ Precarious dimension } \\
\hline $\begin{array}{l}\text { disempowerment and } \\
\text { collective bargaining }\end{array}$ & "collective bargaining" \\
\hline $\begin{array}{l}\text { downsizing and major } \\
\text { organizational restructuring }\end{array}$ & downsizing ${ }^{\mathrm{a}} \mathrm{OR}$ organisational change ${ }^{\mathrm{a}} \mathrm{OR}$ organizational change ${ }^{\mathrm{a}} \mathrm{OR}$ privatization \\
\hline employee involvement & "employee involvement" \\
\hline income wages & $\begin{array}{l}\text { working poor OR low wages OR low income* OR low salary OR inadequate income OR } \\
\text { inadequate wages OR inadequate salary OR insufficient income OR insufficient wages OR } \\
\text { insufficient salary }\end{array}$ \\
\hline job insecurity & 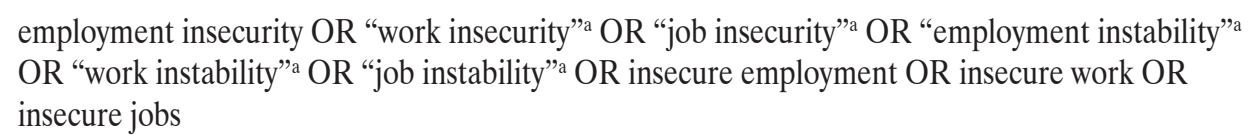 \\
\hline temporariness & $\begin{array}{l}\text { temporary employment OR temporary work OR temporary jobs OR nonpermanent employment } \\
\text { OR nonpermanent work OR non permanent employment OR non permanent work OR non } \\
\text { permanent job OR non-permanent employment OR non-permanent work OR discontinuous } \\
\text { employment OR discontinuous work OR discontinuous jobs }\end{array}$ \\
\hline vulnerability & "worker* vulnerability"a \\
\hline workers' rights & workplace rights ${ }^{\mathrm{a}}$ OR labour union OR labor union OR unionization ${ }^{\mathrm{a}}$ OR unionisation ${ }^{\mathrm{a}}$ \\
\hline working time arrangements & $\begin{array}{l}\text { fixed-term employment OR fixed-term work OR fixed-term jobs OR fixed-term contract OR } \\
\text { fixed term employment OR fixed term work OR fixed term jobs OR fixed term contract OR work } \\
\text { time control OR "worktime control”a OR "work-time control” a OR "inconvenient hours"a OR } \\
\text { parttime employment OR parttime work OR part time employment OR part time work OR part } \\
\text { time jobs OR part time contract OR part-time employment OR part-time work OR part-time } \\
\text { jobs OR part-time contract OR fixed work scheduling OR flexible work scheduling OR over time } \\
\text { employment OR over time work OR over time jobs OR overtime employment OR overtime work } \\
\text { OR overtime jobs OR over-time employment OR over-time work OR over-time jobs OR "long } \\
\text { working hours"a }\end{array}$ \\
\hline multidimensional & $\begin{array}{l}\text { precariousness }{ }^{a} \text { OR nonstandard employment OR nonstandard work OR nonstandard jobs OR } \\
\text { non standard employment OR non standard work OR non standard jobs OR "non-standard } \\
\text { employ*”a OR “non-standard work" }{ }^{*} \text { ”a } \text { OR "non-standard job"”a OR atypical employment OR } \\
\text { atypical work OR atypical jobs OR contingent employment OR contingent work OR contingent } \\
\text { jobs OR flexible employment OR flexible work OR flexible jobs OR precarious work OR } \\
\text { precarious employment OR precarious jobs }\end{array}$ \\
\hline \multicolumn{2}{|l|}{ Outcome } \\
\hline mental health & mental health OR mental disorders \\
\hline Country & $\begin{array}{l}\text { austria OR belgium OR bulgaria OR croatia OR cyprus OR czech republic OR denmark OR } \\
\text { estonia OR finland OR france OR germany OR greece OR hungary OR ireland OR italy OR } \\
\text { latvia OR lithuania OR luxembourg OR malta OR netherlands OR poland OR portugal OR } \\
\text { romania OR slovakia OR slovenia OR spain OR sweden OR "united kingdom" OR norway OR } \\
\text { switzerland OR "european union" OR Europe OR "great britain" }\end{array}$ \\
\hline
\end{tabular}

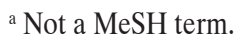




\section{Quality control and data mining}

The quality of the studies included was assessed using the National Heart, Lung and Blood Institute's Quality Assessment Tool for Observational Cohort and Cross-Sectional Studies [25]. Based on the 14 questions of the quality assessment tool, the reviewers assigned each article a global evaluation indicating good quality, fair quality or poor quality. Longitudinal articles were assessed as poor quality if they had $\geq 4$ negative answers, and as fair quality if they had 2-3 negative answers. Cross-sectional articles were evaluated as poor quality if they had $>1$ negative answer (except in items 5, 8 and 12), and as fair quality if they had 1 negative answer (except in items 5, 8 and 12). Articles graded as poor quality were excluded from the review. The studies included were synthesized using a data extraction table including:

- general characteristics (author[s], year of publication),

- methodological factors (reference population, samples, exposure variables, outcomes and study design),

- results (types of statistical analysis, measures of association, direction and strength of the evidence),

- conclusions.

\section{RESULTS}

The selection process is summarized in the study selection flow diagram in Figure 1. The database search retrieved a total of 1522 articles, this number being reduced to 1301 by removing duplicates. Of these, 1154 were rejected after reading the title and abstract, and 7 articles were manually added after consulting the references cited in previous reviews in the field. This yielded 154 articles to be screened based on the full text. Of these, 97 did not meet the inclusion criteria, mainly because they did not assess the association between precarious employment and mental health, the explanatory variable did not measure precariousness, or the dependent variable did not refer to mental health, while 3 studies were of poor quality (Table 2 - full results of the quality control analysis). Hence, 54 articles were in-

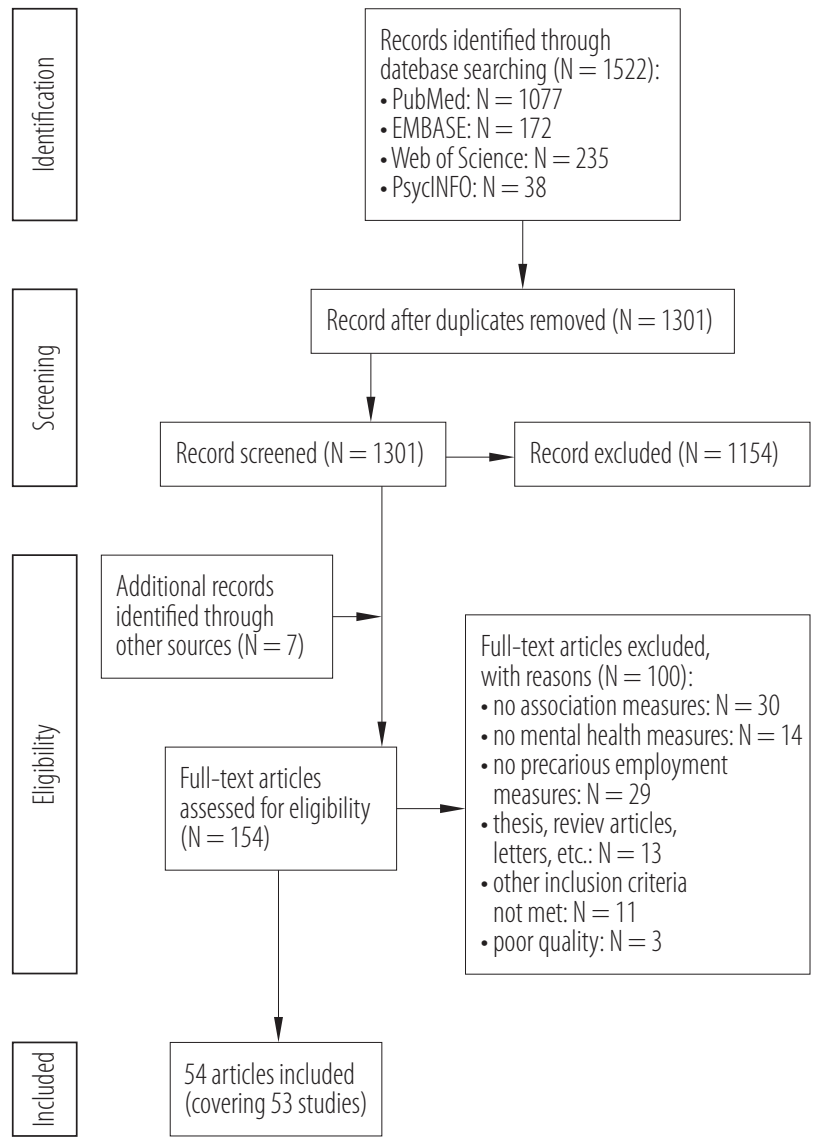

Figure 1. Selection of the studies on the association between precarious employment and mental health published in 2010May 2018, based on data concerning the European Union, Norway, Island, Liechtenstein and Switzerland - a flow diagram

cluded in the review, corresponding to 53 studies, as 1 study was described in 2 papers [26,27].

\section{Characteristics of the studies}

The main characteristics of the studies are presented in Table 3. Most of the studies had a (single) cross-sectional design, except for 6 repeated cross-sectional studies and 12 longitudinal studies. They had been conducted in Southern Europe (12 in Spain), 12 in Nordic countries, 11 in Western Europe, and 2 in Central Europe, while a total of 12 studies simultaneously analyzed data from various European countries. More than half of the studies were published in 2015 or later. The data analyzed were collected in 


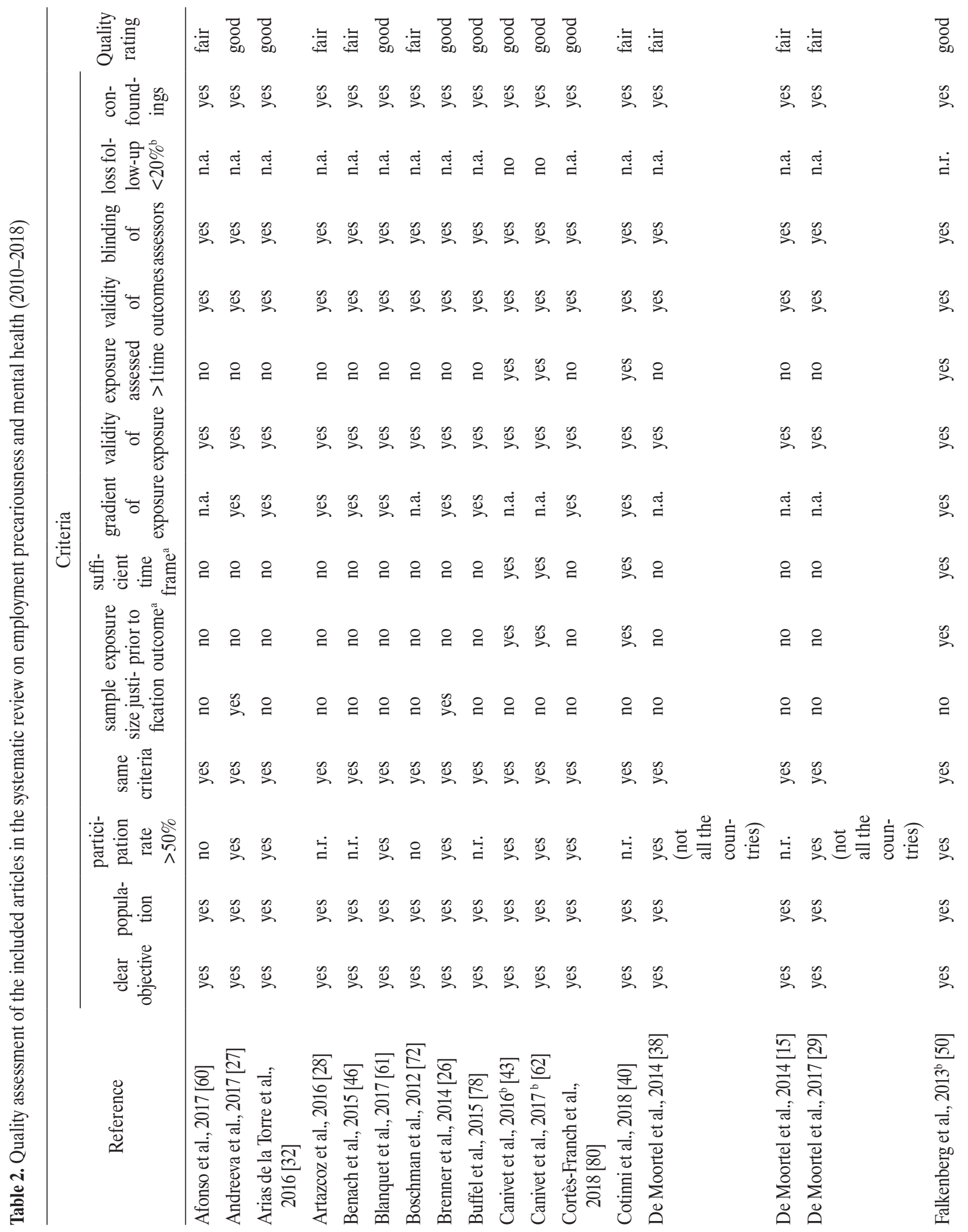




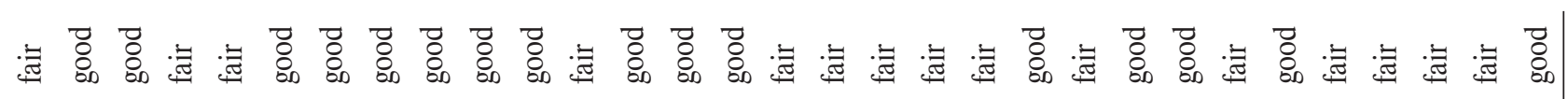

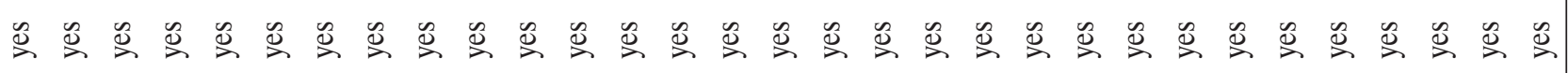

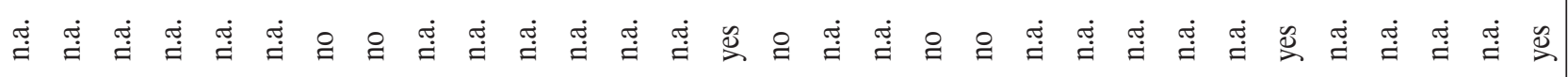

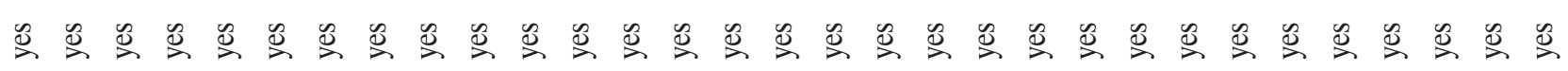

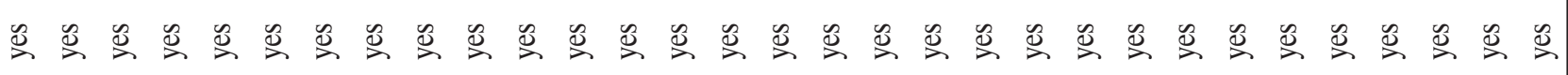
总

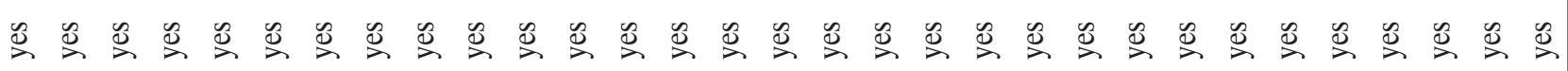

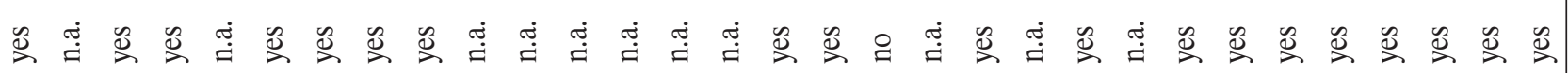

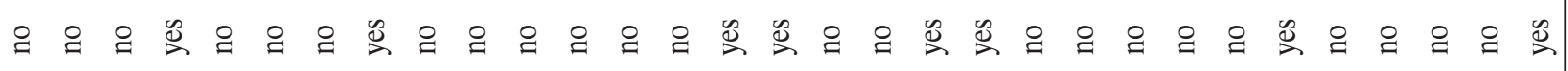

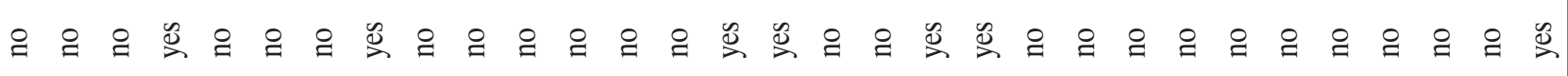

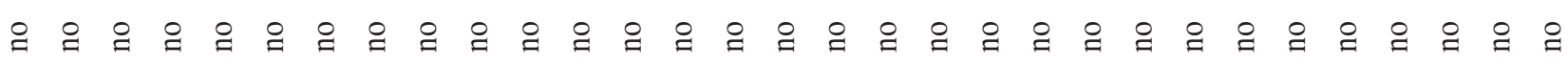

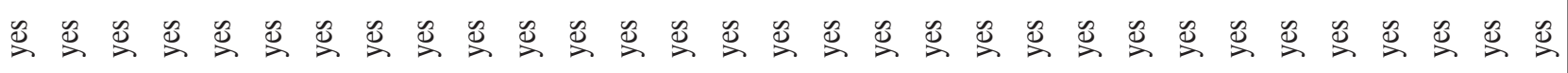

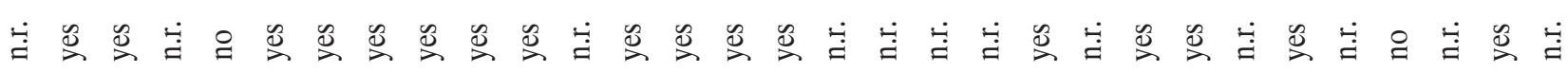

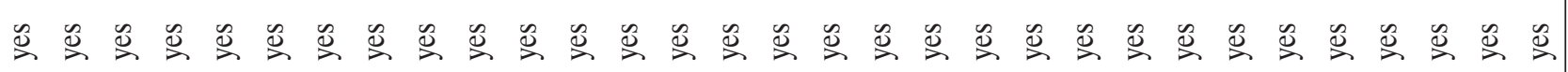

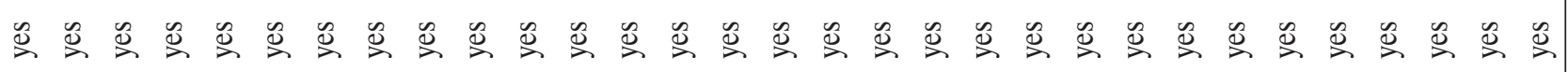

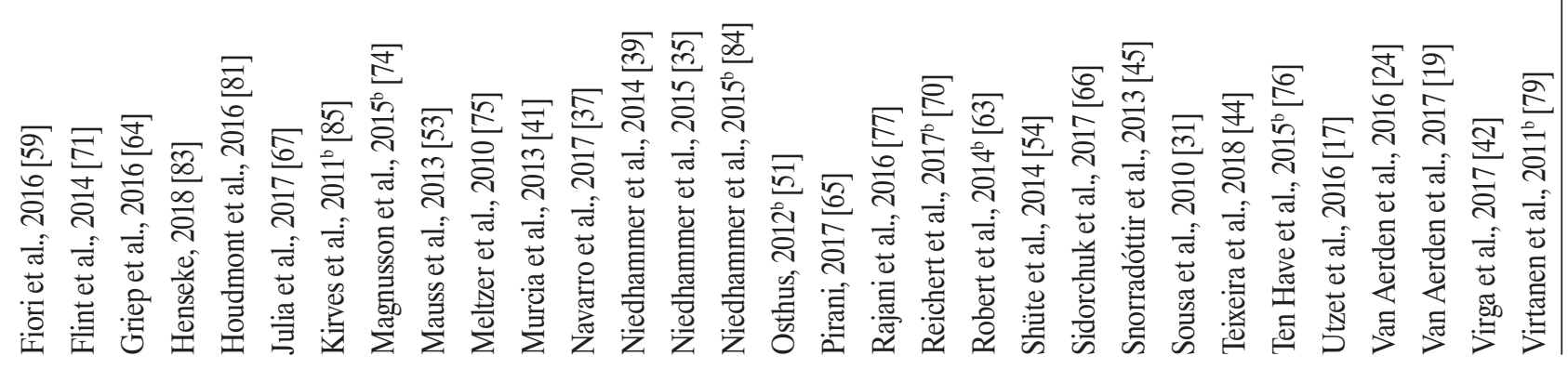




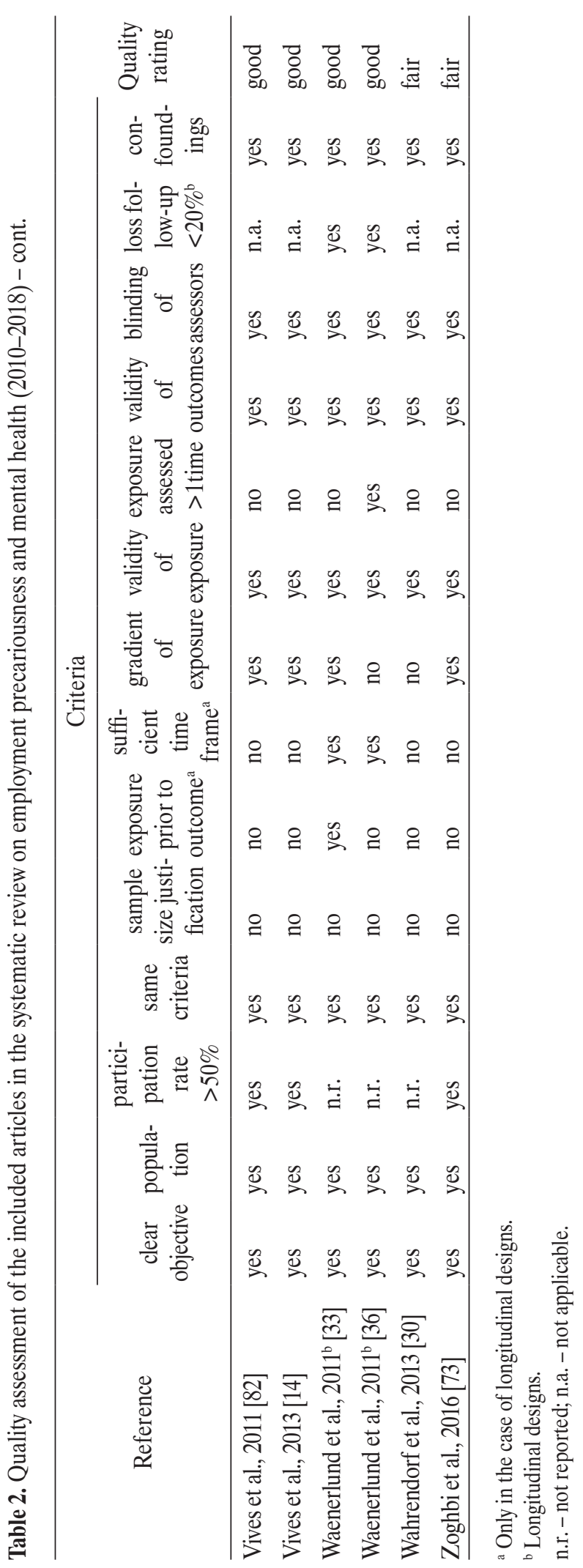

1995-2014 (except for some longitudinal analysis including earlier data), and 35 studies used data collected since 2009. Most studies analyzed representative samples of the general working population.

\section{Dimensions of precarious employment and its association with mental health}

Results are organized as a function of the dimensions of precarious employment analyzed (Table 3 ). The exposures studied were:

- job insecurity $(\mathrm{N}=20,37.7 \%)$;

- temporariness $(\mathrm{N}=12,22.6 \%)$;

- multidimensional approach $(\mathrm{N}=10,18.9 \%)$, which was in turn subdivided into the job quality approach, the EPRES approach, and other multidimensional approaches;

- working time arrangements $(9,17.0 \%)$;

- downsizing and major organizational restructuring $(5,9.3 \%)$,

- income wages $(4,7.5 \%)$.

In all the studies, the assessed mental health measures were subjective (psychological distress, depression, anxiety, and mental health symptoms) and were obtained using instruments such as the General Health Questionnaire, the 36-item Short-Form Health Survey, the 5-item Mental Health Inventory and the Hospital Anxiety and Depression Scale.

Among the studies that analyzed organizational restructuring processes, 3 found that individuals who kept their jobs were at a greater risk of depression, anxiety and psychological distress (especially when such processes were reactive, and workers were not informed in advance). In 2 longitudinal studies, this effect was short-term. Out of the 4 studies assessing salaries, 3 found a significant association between a low salary and poor mental health in both sexes. Among the 20 studies that evaluated perceived job insecurity, 18 found a significant association with depression, anxiety and mental health problems, also in both sexes. All 12 studies assessing temporary contracts 


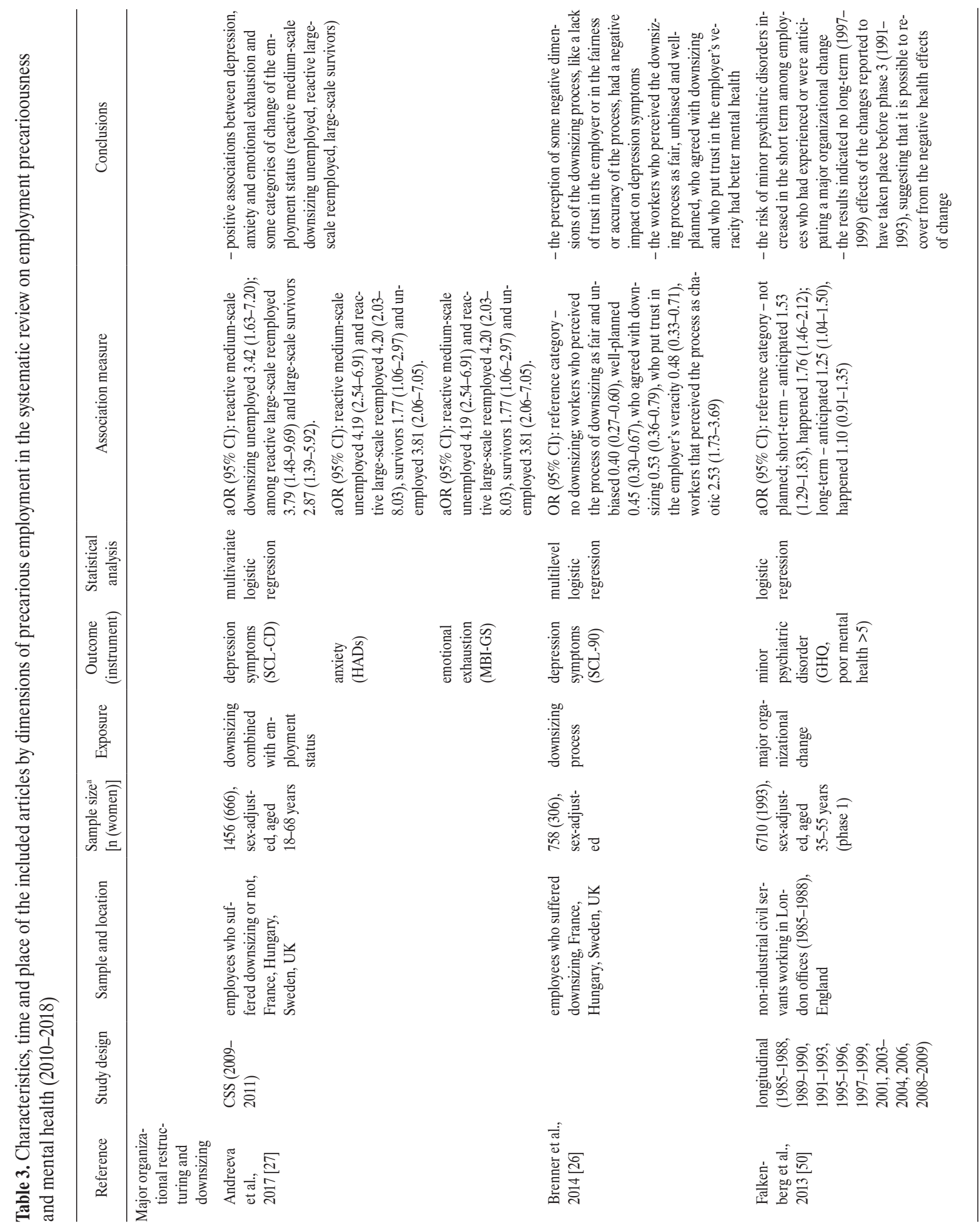




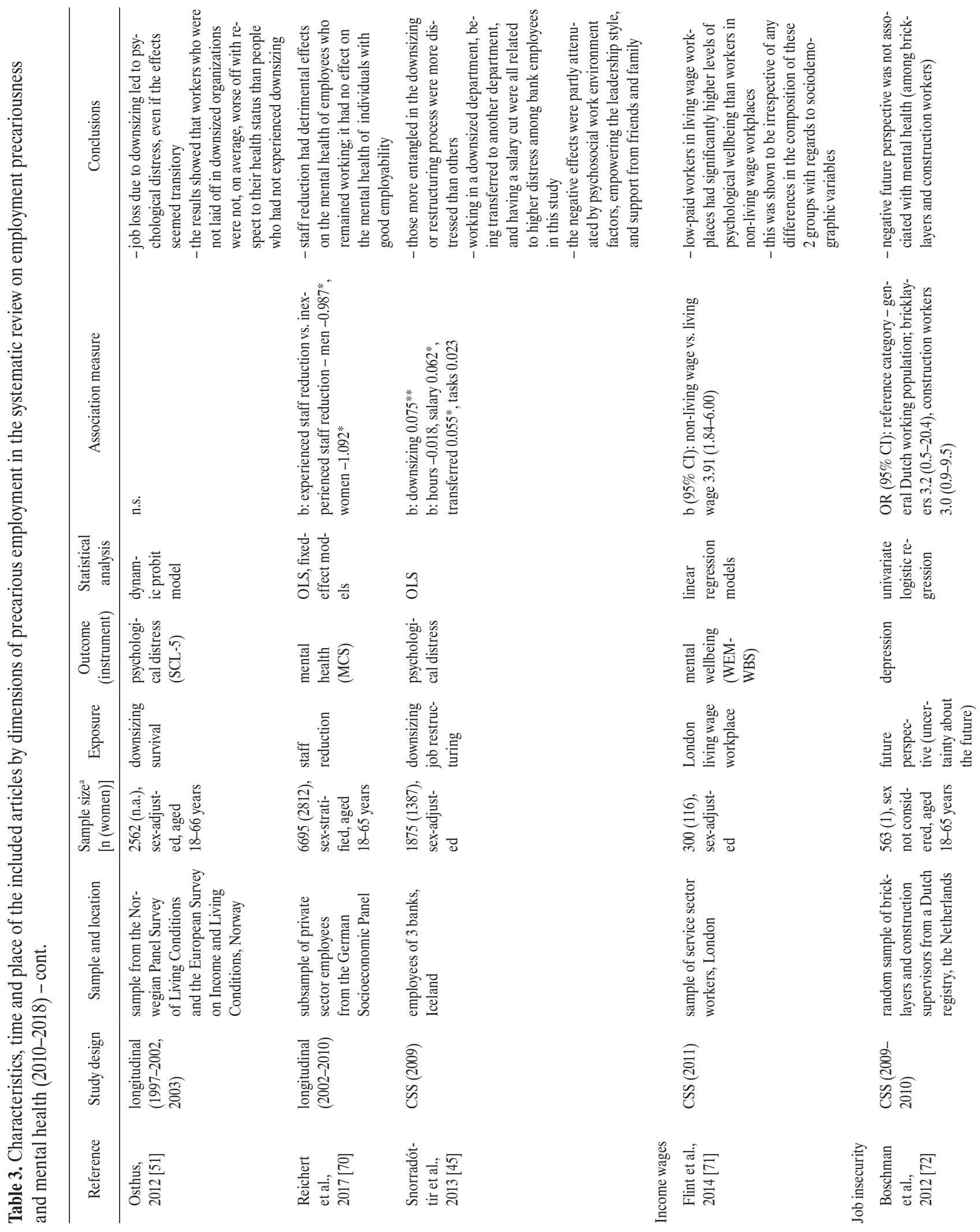



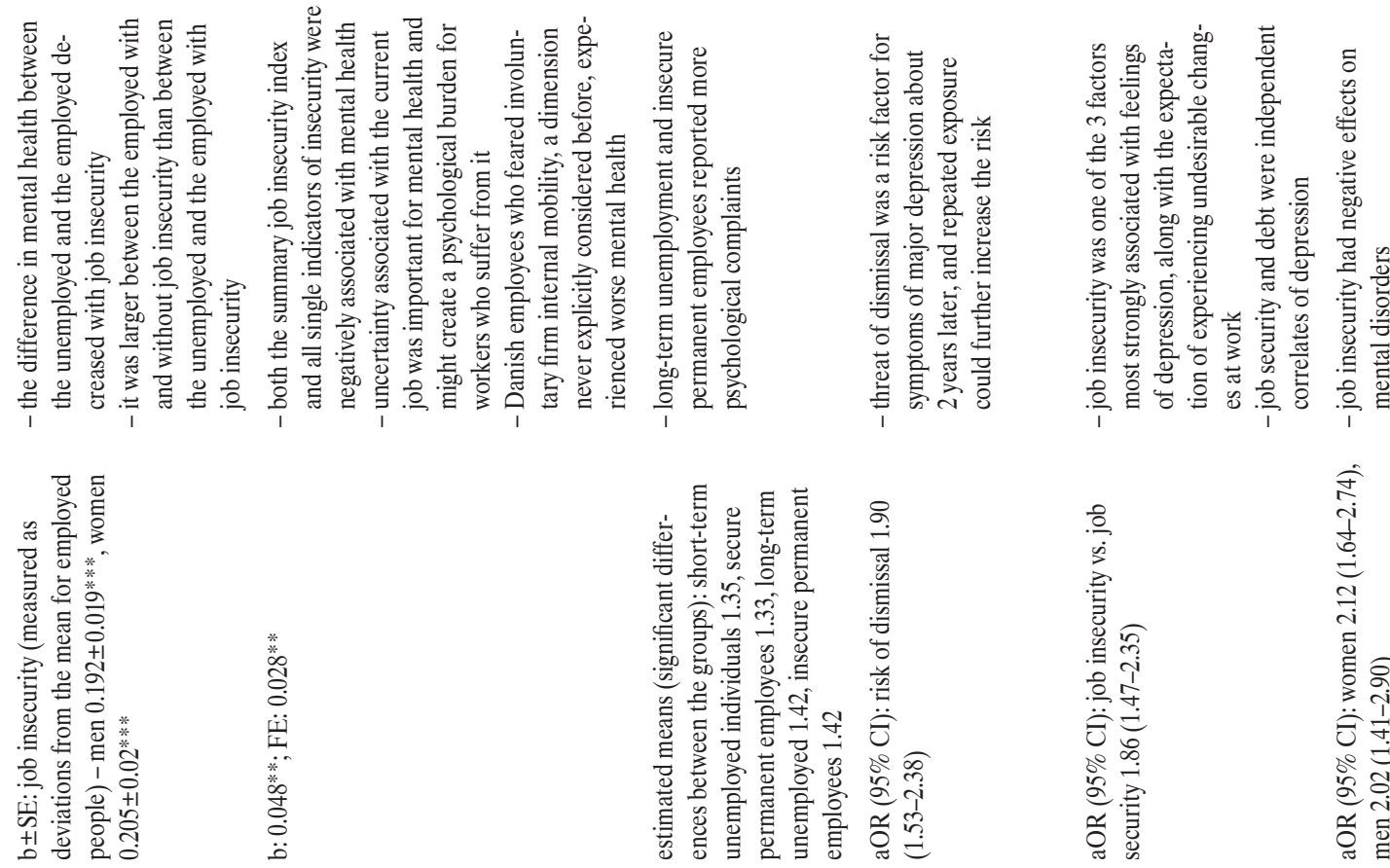

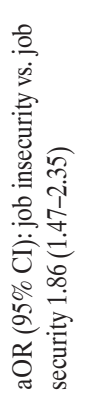
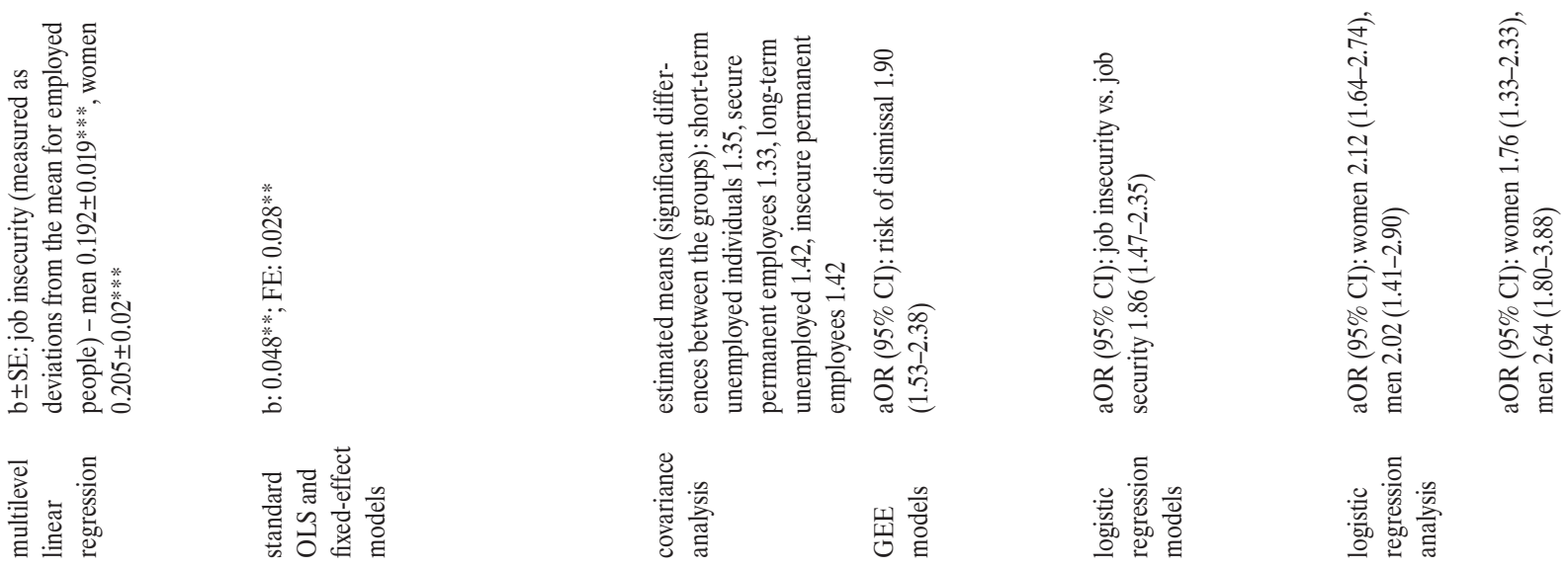

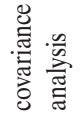

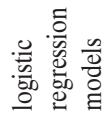

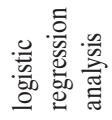

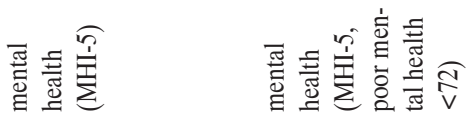

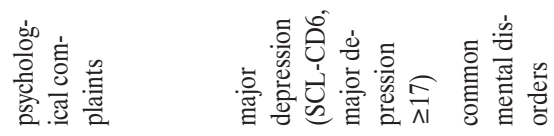

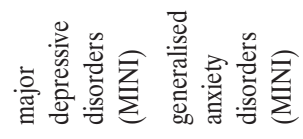

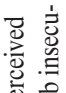

혼을 즐

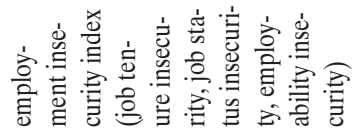

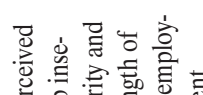

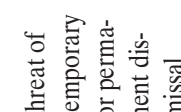

竞

言

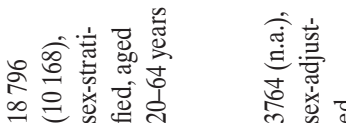

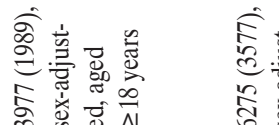

在离

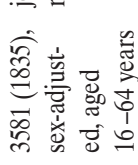

으를
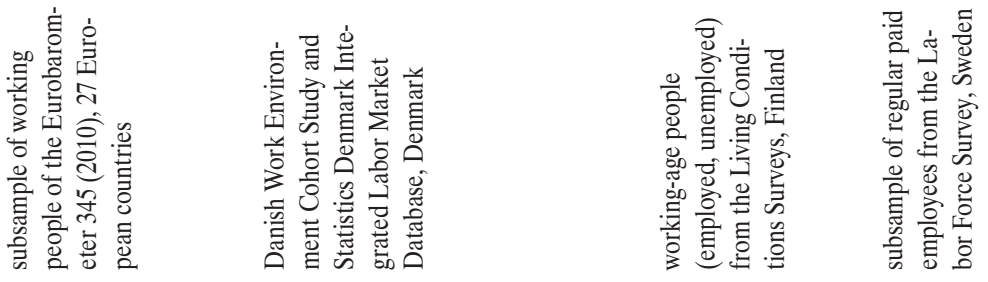

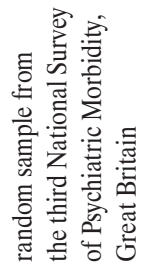

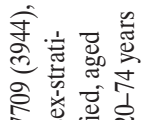

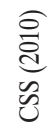

플

悉

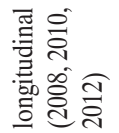

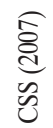

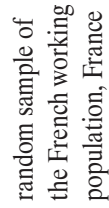

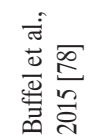

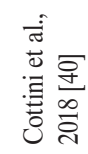

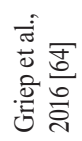

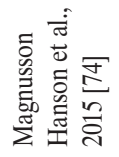

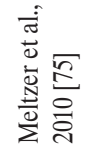

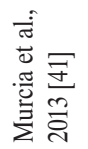




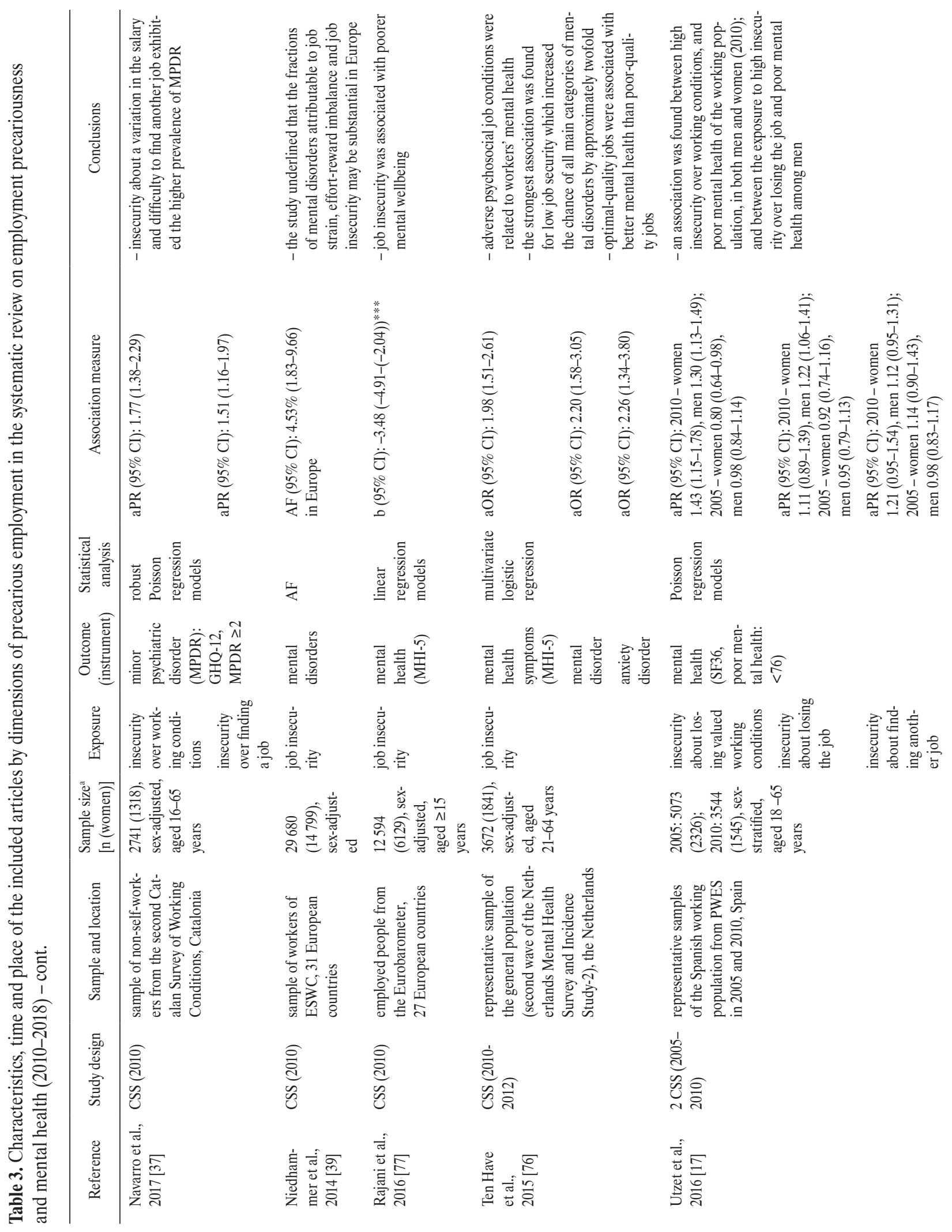




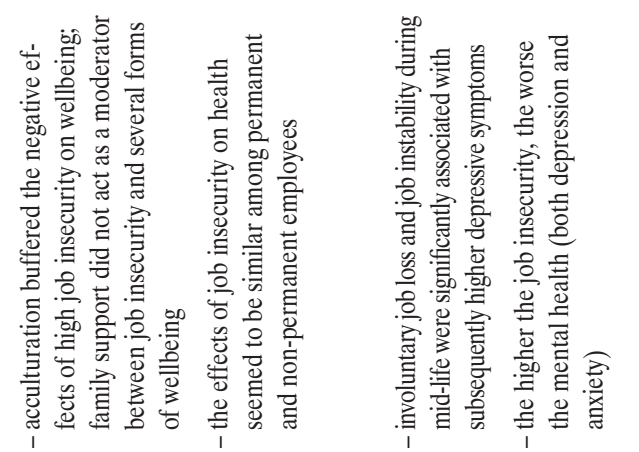

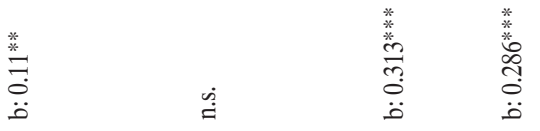

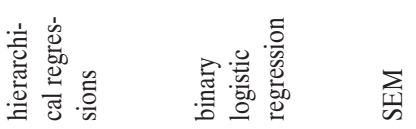

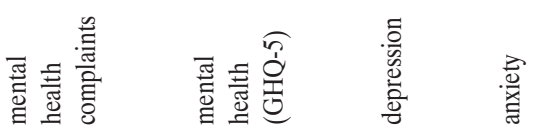

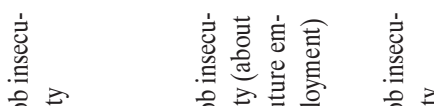

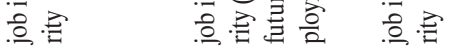

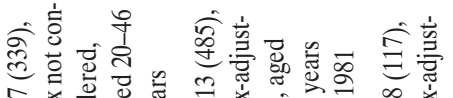

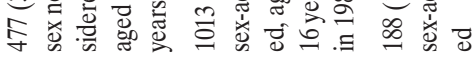
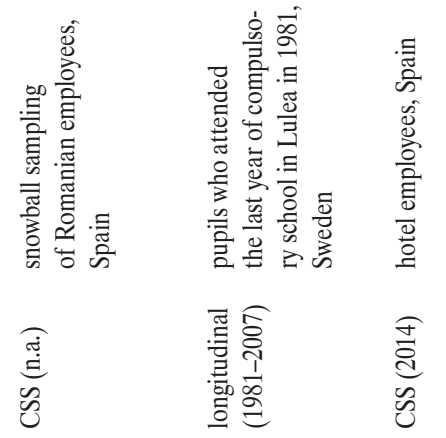

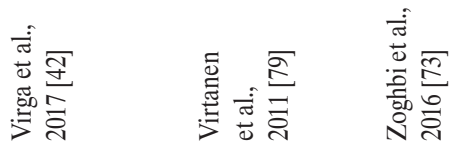

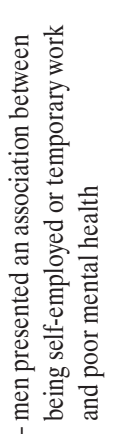

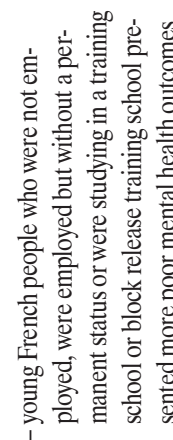

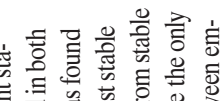

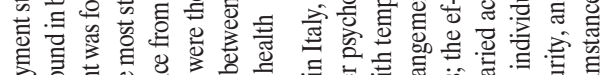

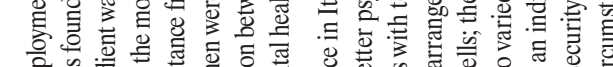

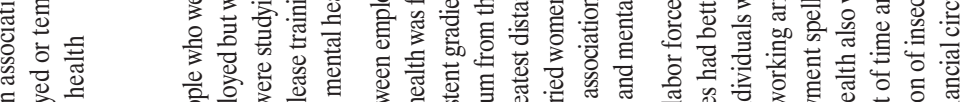

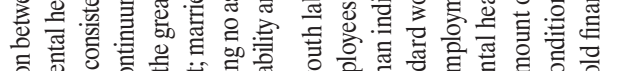

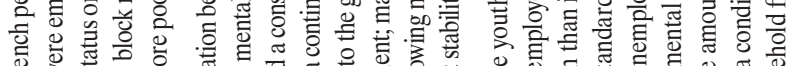

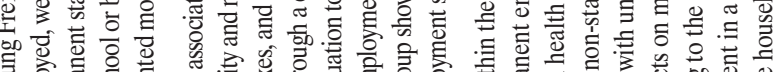

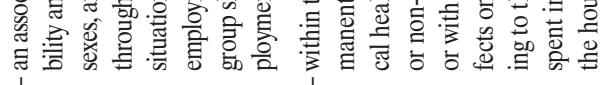

$$
\text { 言 }
$$

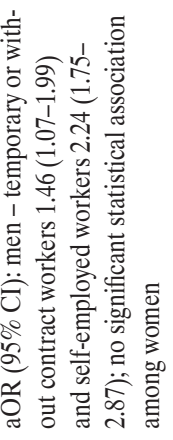

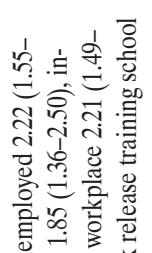

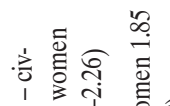

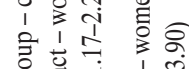

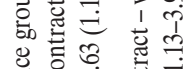

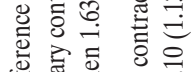

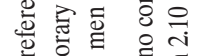

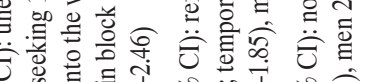

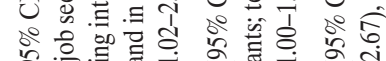

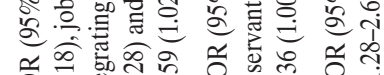

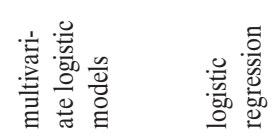

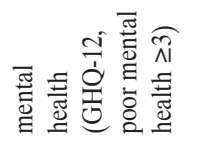

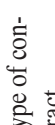

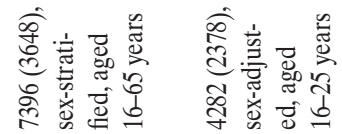

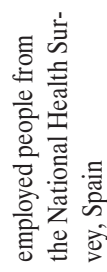

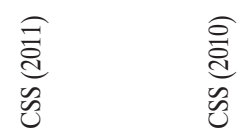

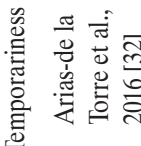

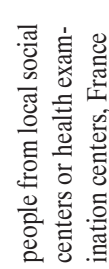

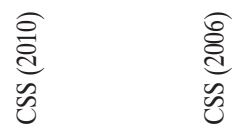

高

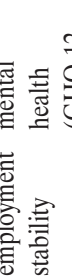

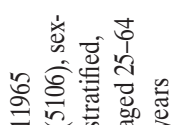

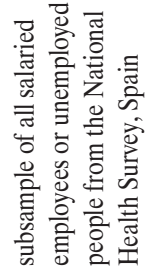

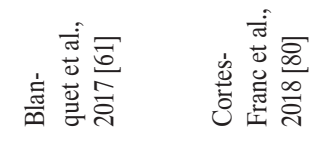

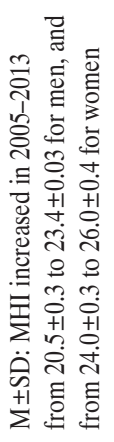

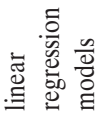

焉焉突

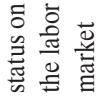

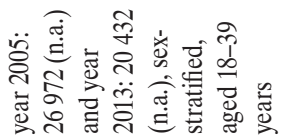

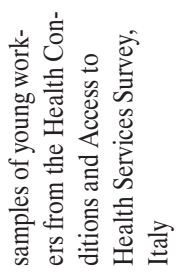

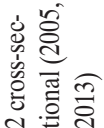

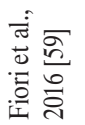




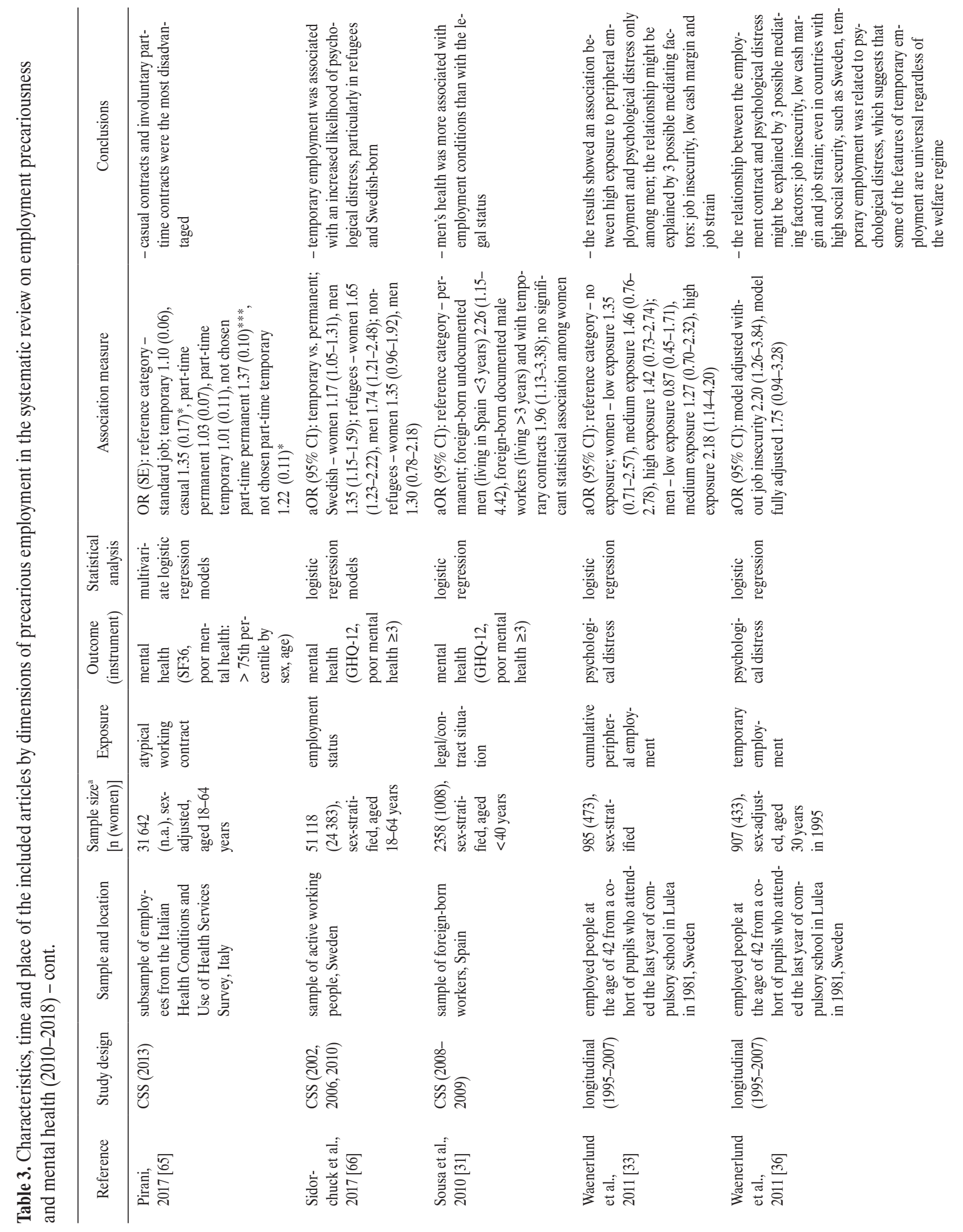



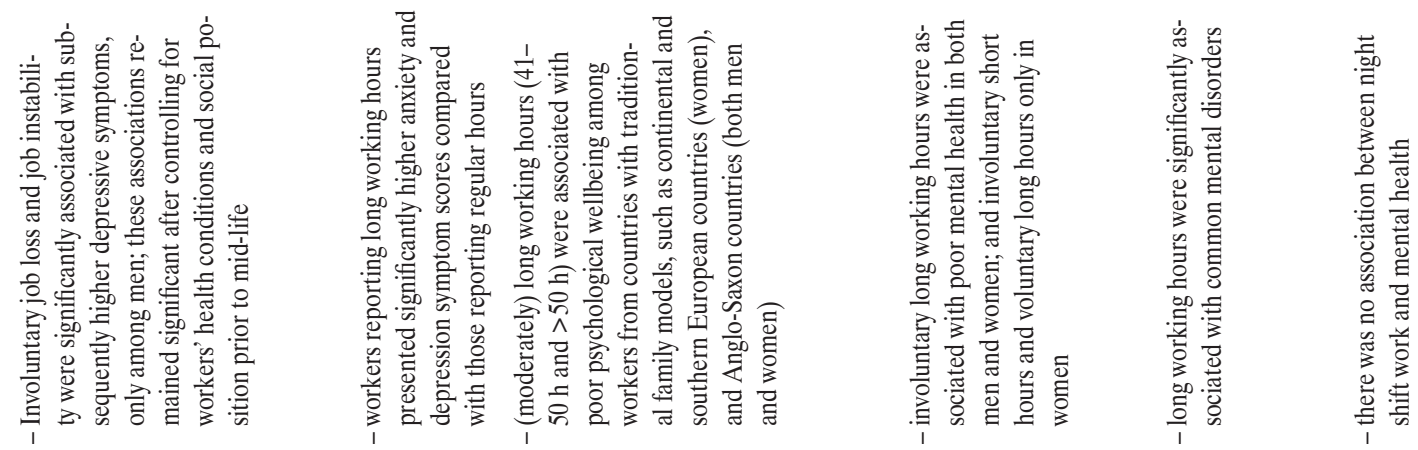

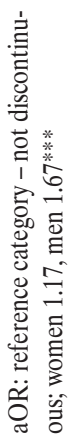

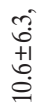

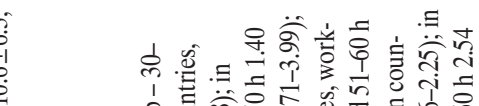

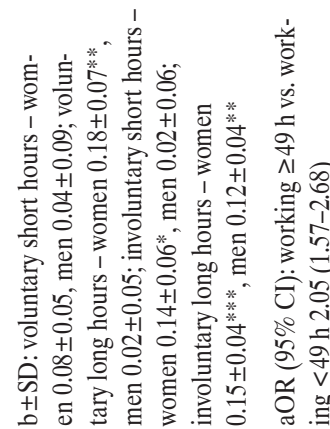

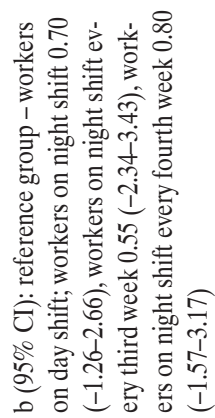

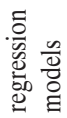

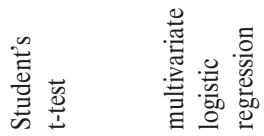

总急

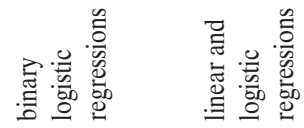

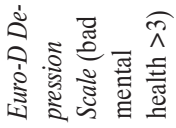

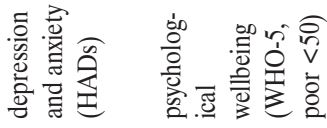

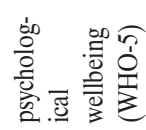

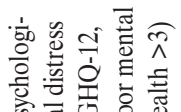

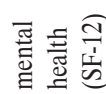

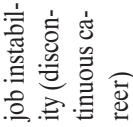

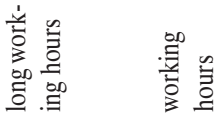

辛高

岁战

吾蓄

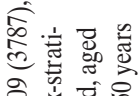

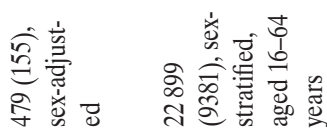

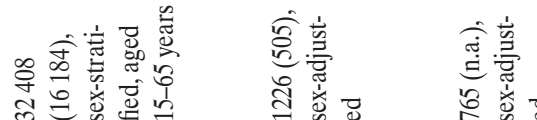

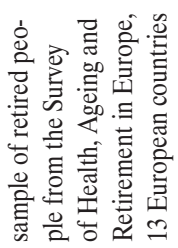

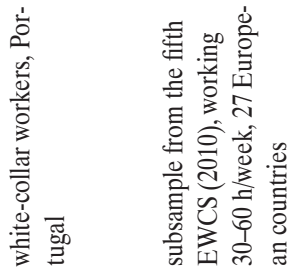

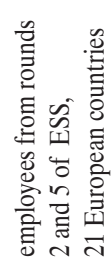

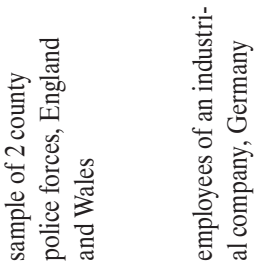

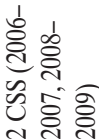

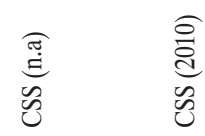

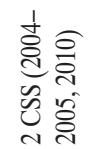

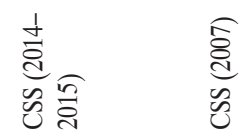

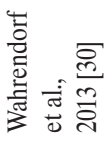

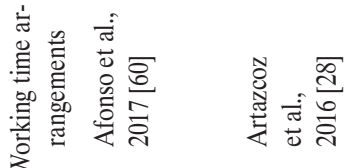

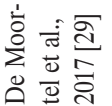

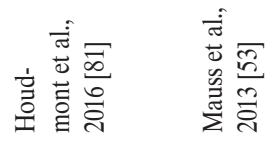




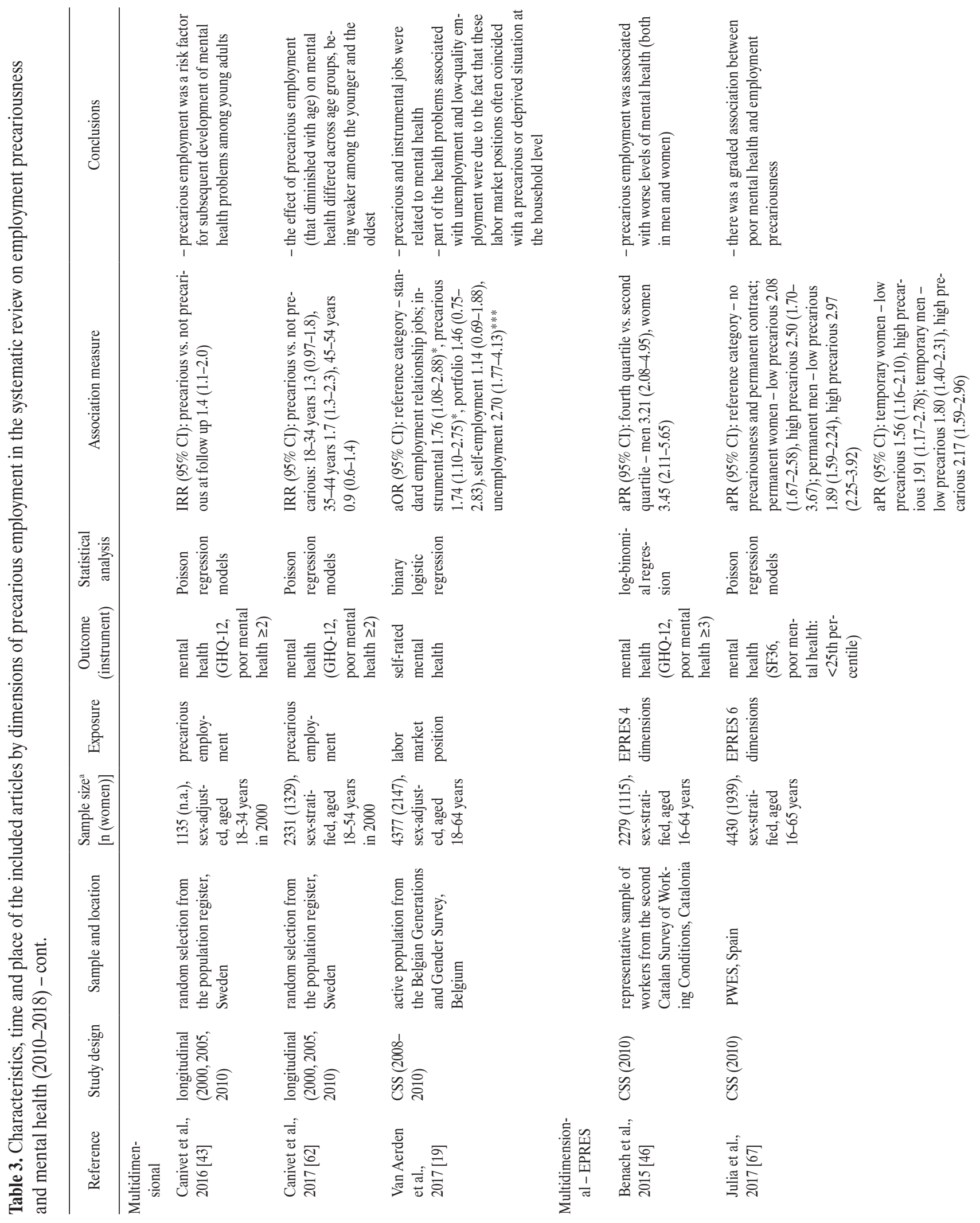



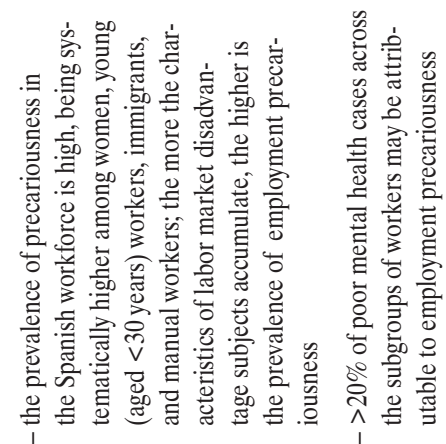

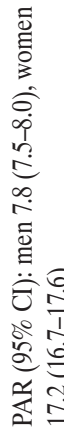

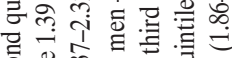

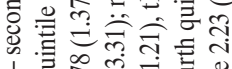

1 絜宁

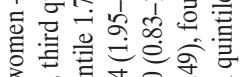

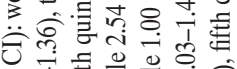

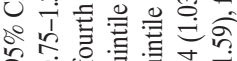

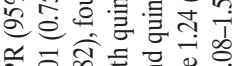

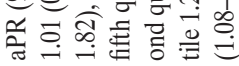

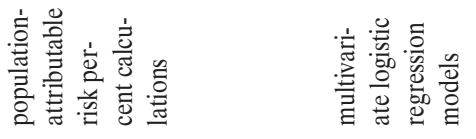

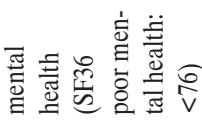

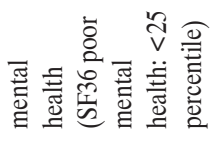

号言

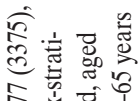

点离总造

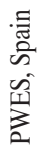

芯

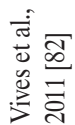

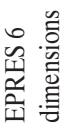

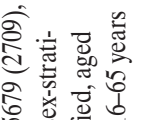

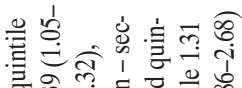
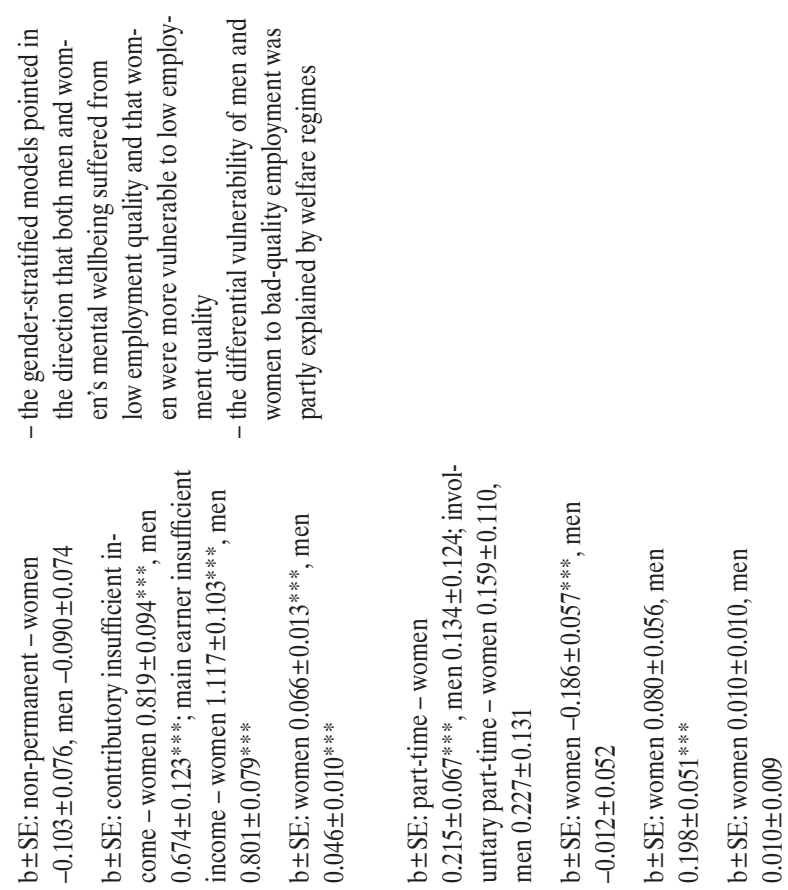

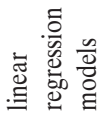

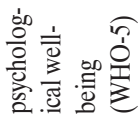

兽兽.

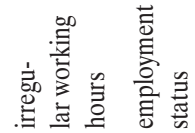

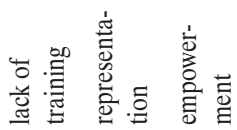

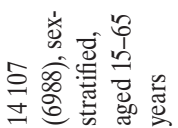

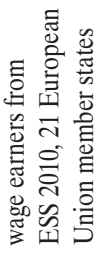

高

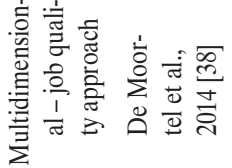




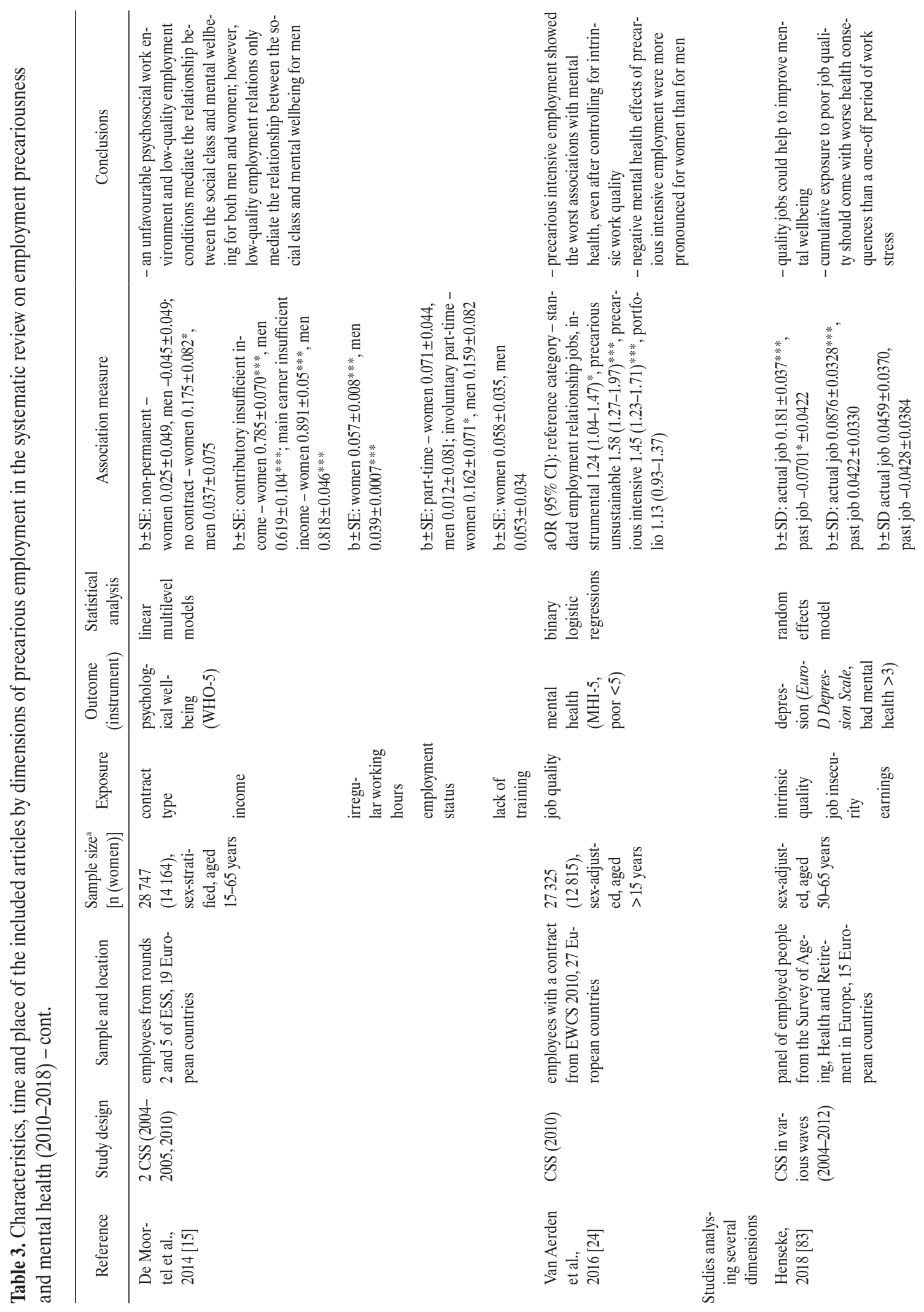



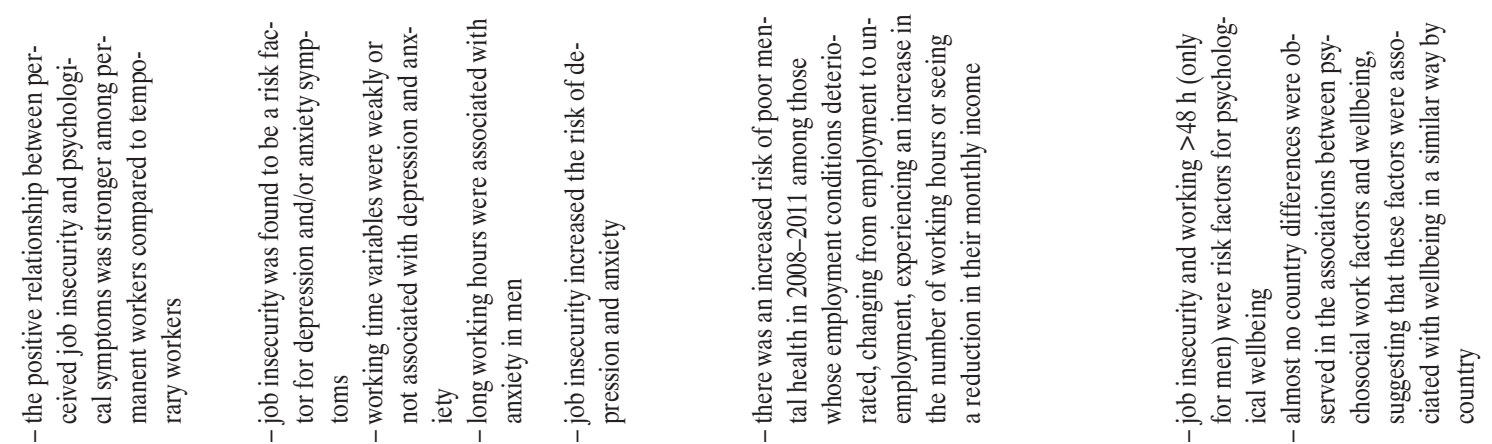

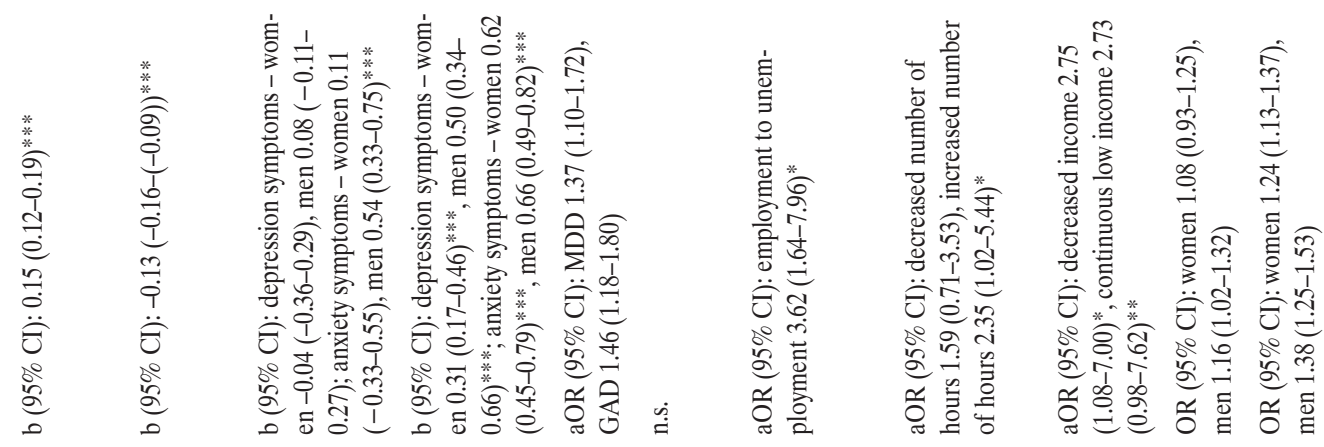

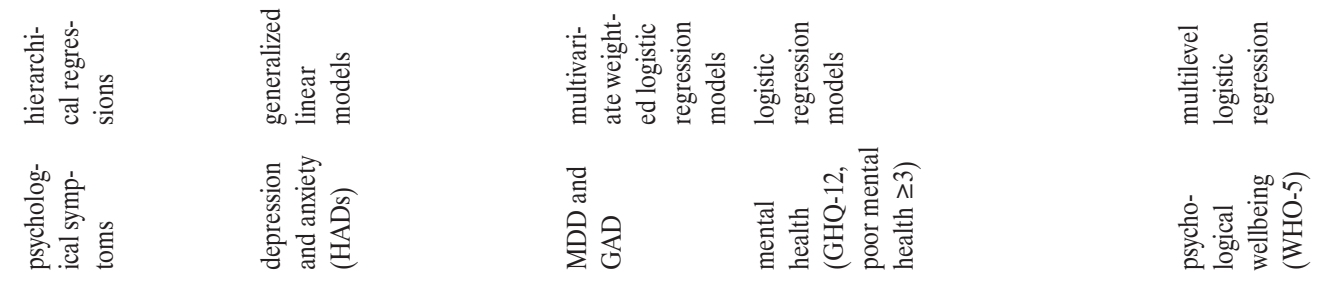

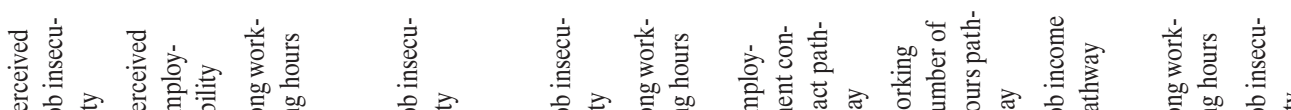

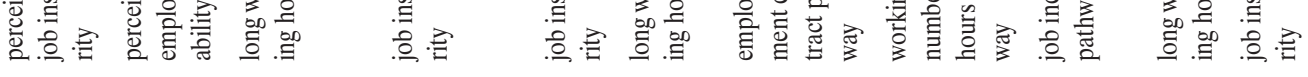

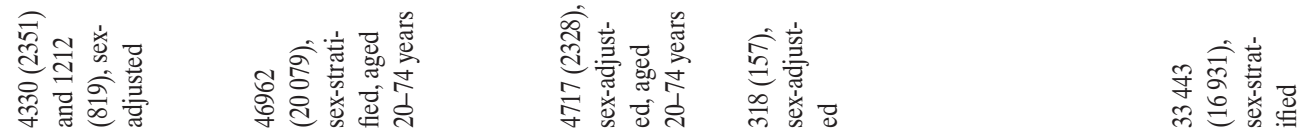

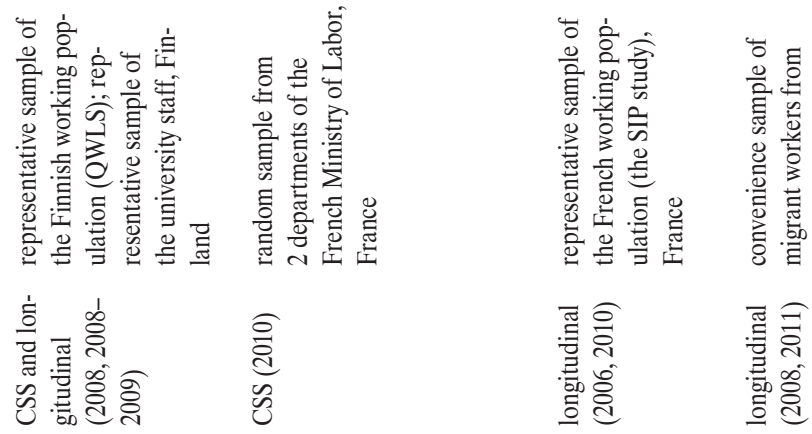

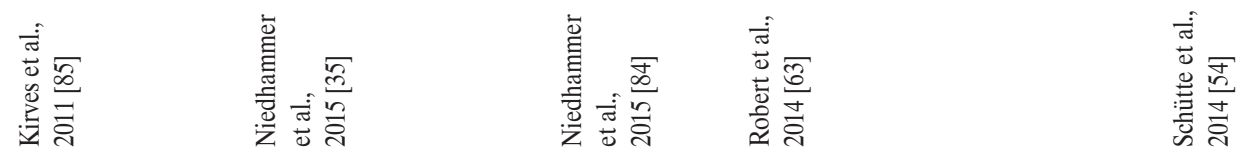




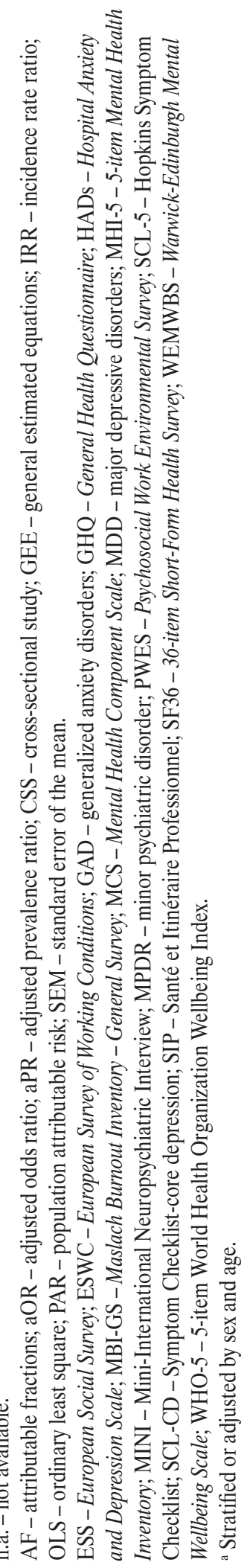

found significant associations, 9 in both sexes and 3 mainly in men. Out of the 9 studies analyzing working time arrangements, 5 found working long hours to be associated with poor mental health, depression, psychological wellbeing and common mental disorders (1 of them specifying whether the long hours were worked on a voluntary basis or not) in both sexes, and 2 studies found such associations only in the case of men. Finally, among the 10 studies that analyzed forms of precarious employment, which was specifically addressed as a multidimensional concept, all found significant associations with depression in both sexes (2 studies mainly in women), as well as with poor general mental health and psychological distress.

Summarizing the results in terms of sex stratification, only $22(41.5 \%)$ studies presented the results this way. Regarding stratification by sex and dimension, it was carried out in $7(58.3 \%)$ of the studies assessing temporariness, of which $7(70.0 \%)$ used a multidimensional approach, $2(22.2 \%)$ analyzed working time arrangements, $5(25.0 \%)$ described job insecurity (3 included in the job insecurity section and 2 in the several dimensions section), 1 (20.0\%) focused on downsizing, while no study assessed job income. Among them, 11 associations assessed in 10 of the articles identified differences between men and women but the findings were not consistent. Two studies $[28,29]$ concluded that women were more vulnerable to the consequences of long working hours, and 1 study [15] to the type of contract (no contract) and to part-time work. The other 7 articles found that temporariness (measured with different types of variables) [30-33], insecurity about finding a job [17] and working $>48 \mathrm{~h} /$ week [34,35] were associated with poorer mental health among men, but not among women.

\section{Causal pathways}

Among the studies that reported significant associations, 21 described some of the potential pathways that associate precarious employment with mental health indicators 
Table 4. Articles by exposure dimension and hypothesized pathways in the systematic review on employment precariousness and mental health (2010-2018)

\begin{tabular}{|c|c|c|c|c|c|c|}
\hline \multirow{3}{*}{$\begin{array}{l}\text { Exposure } \\
\text { dimension }\end{array}$} & \multirow{3}{*}{$\begin{array}{l}\text { Studies } \\
\text { [n] }\end{array}$} & \multicolumn{5}{|c|}{$\begin{array}{l}\text { Hypothesized pathway } \\
{[\mathrm{n}]}\end{array}$} \\
\hline & & \multirow{2}{*}{ lack of control } & \multirow{2}{*}{ breadwinner model } & \multicolumn{2}{|c|}{ deprivation model } & \multirow{2}{*}{$\begin{array}{c}\text { precarious job } \\
\text { linked to worse } \\
\text { working conditions }\end{array}$} \\
\hline & & & & financial benefits & latent functions & \\
\hline Downsizing & 6 & $1[27]$ & $1[70]$ & & & \\
\hline Income & 4 & $1[63]$ & & $1[63]$ & $1[63]$ & \\
\hline Job insecurity & 20 & & $2[17,78]$ & & $1[64]$ & \\
\hline Temporariness & 12 & $1[63]$ & $3[32,59,80]$ & $3[36,59,63]$ & $2[63,65]$ & $1[66]$ \\
\hline $\begin{array}{l}\text { Working time } \\
\text { arrangements }\end{array}$ & 9 & $2[29,63]$ & $3[28,29,60]$ & $1[63]$ & $1[63]$ & \\
\hline Multidimensional & 10 & $1[67]$ & $4[14,15,38,67]$ & $2[62,67]$ & & $3[24,43,67]$ \\
\hline
\end{tabular}

${ }^{a}$ Includes double counting.

(Table 4). When studies proposed several different pathways, double counting was unavoidable, and findings for multiple-category studies were separated for each category. These can be grouped into 5 different types of explanation:

- the breadwinner model (12 studies),

- the economic deprivation model (5 studies),

- the association between exposure to precariousness and poorer working conditions (4 studies),

- the lack of control over one's work situation (4 studies),

- and the latent deprivation model (3 studies).

Considering the above observations, a conclusion can be drawn that causal pathways are more often discussed in studies analyzing temporariness ( 7 out of $12,58.3 \%$ ), income ( 3 out of $4,75.0 \%$ ) and multidimensional approaches (7 out of $10,70.0 \%$ ) than in those assessing working time arrangements ( 3 out of 9, 33.3\%) downsizing ( 2 out of $6,33.3 \%$ ) and job insecurity ( 3 out of 20 , $15.0 \%)$. In most of the articles, pathways were proposed in a hypothetical way, and only in 2 of them $[24,36]$ these pathways were tested by introducing in the statistical models control variables related to these pathways.

\section{Macro- and micro-level factors}

More than half $(36,66.7 \%)$ of the studies analyzed data past 2008 (in repeated cross-sectional and longitudinal designs, at least 1 wave or follow-up). Among these, 13 (24.1\%) described the context of the economic, political and social crisis; although only 5 conducted a more in-depth description of its influence in the association of precarious employment and mental health [17,27,28,37,38]. One study analyzing downsizing [27] concluded that the exposure to layoffs during the economic crisis had probably made the survivors exceptionally vulnerable to the stress of potential reactive downsizing. Two studies analyzing employment quality [38] and working time arrangements [28] showed that part-time employment and working moderately long hours were positively associated with poor female mental wellbeing in southern European countries. Both articles interpreted the results in the crisis context in which there was an increase in the participation of women in the labor market due to the weakening household economy. Two studies analyzing job insecurity $[17,37]$ explained the high association between this dimension and mental health after 2008 in the context of high unemployment and job destruction after the crisis. 
Twelve (22.2\%) studies focused on various European Union member states. Including double counting, 5 (41.7\%) of them analyzed job insecurity, 4 (33.3\%) used a multidimensional approach to precarious employment, $3(25.0 \%)$ were based on working time arrangements, $1(8.3 \%)$ focused on downsizing and $1(8.3 \%)$ on temporariness. Most of these studies (as many as 9) performed statistical analysis adjusting for context variables. The rest of the studies conducted analyses stratified by the state (1 study [39]) or the type of the welfare regime (2 studies [28,38]). The former presented significant differences between countries for the fractions of mental disorders. The last 2 concluded that the (differential) vulnerability of men and women to precarious employment could be partly explained by welfare regimes. Specifically, the association was stronger in countries with traditional family models, deregulated labor markets and poor policy models balancing family life and work.

Finally, 7 (13.0\%) studies considered the role of social and family support for workers in the association. In 2 cases [40,41], it was introduced as a confounder variable in the regression models and no further explanation was given. In 2 studies [42,43], social support variables were found to have no impact on the association, while the other 3 studies demonstrated that social and family support as well as the household situation decreased the impact of precarious employment (1 study, [19]), income and employment quality [44] and restructuring processes (1 study, [45]) on mental health.

\section{DISCUSSION}

The objectives of this paper were to review and summarize the evidence assessing the association between precarious employment and mental health. The authors found 53 studies of sufficiently good quality and reporting relatively recent data that met the inclusion criteria. They analyzed different aspects of precarious employment and almost all found statistically significant associations with mental health.

During recent decades, research on precarious employment and its consequences for workers' health has spread $[4,16]$.
Despite the consensus regarding the need to adopt a multidimensional perspective, there is still a lack of standardized terminology, from univariate approaches to various multidimensional conceptualizations $[9,24,38,46]$. For this reason, during the search stage of this review, attempts were made to include a large range of terms related to precarious employment, in order to understand the full extent of this phenomenon.

As it has been presented, the main dimensions studied in the literature continue to be job insecurity and temporariness, which are indeed important issues but they merely provide a partial view of the reality [7]. Job insecurity concerns the possibility of losing one's job in the coming months or the difficulty of finding a new job after becoming unemployed; however, some authors also address job insecurity in relation to the worsening of working conditions in the current employment [17,37]. In the first case, job insecurity is more closely related to employment continuity, while in the second, it is more about a loss of control over one's work, whether in terms of working conditions, or the nature or pace of work. Almost all the studies analyzed found an association between exposure to job insecurity and mental health problems, which is consistent with the findings of previous research [47].

Temporary jobs represent, by nature, a lack of security concerning employment continuity. However, temporary workers are not only insecure about their future employment; they also tend to face the worst working conditions [48] and have fewer opportunities for training and professional development, and less information regarding their work environment [12], among other issues. Such mechanisms may explain the finding that nearly all of the studies detected associations between having a temporary contract and mental health problems, in line with a previous review [2]. Nevertheless, factors playing a moderating role, such as perceived job insecurity, motivational aspects (voluntariness) and the workers' social context should be taken into consideration in order to fully understand the mental health consequences of fixed-term contracts [49]. 
The papers studying downsizing establish that there is an association between such processes and workers' mental health although Falkenberg et al. [50] and Osthus [51] concluded that the impact on mental health was reversible. These findings are in line with other reviews [2]. An organizational restructuring process implies the threat of being made redundant or at least some uncertainty regarding employment continuity, which represents a significant source of stress [27]. In order to reduce the impact, some authors have recommended embarking on restructuring processes in a strategic way and ensuring that all stakeholders are informed [26,27].

The studies concerning working time arrangements, especially related to long working hours, are not conclusive. This finding is consistent with a recently published review [52], which found a minor association. Two of the papers, one studying work schedule assignment [53] and the other long working hours [35], found no association, and the 2 analyzing long working hours found such association only among men [35,54]. Unlike the previously discussed indicators, working time arrangements are more closely related to control over one's work than uncertainty [29]. This dimension of precariousness is strongly associated with the willingness to work long hours and, as established in the effort-reward model [55], with remuneration for the hours worked [29], though it is also associated with gender and the social structure in which workers operate, the welfare state, labor market regulations, social norms and family responsibilities [28,29]. Further, workers' willingness to work long hours may not be real when there is external pressure to do so, in order to get a wage that would cover their social and family needs.

Finally, the results of multidimensional approaches show a clear association with mental health. In the last 8 years, a growing number of multidimensional conceptualizations of precarious employment have emerged. This is a more comprehensive approach to the reality, describing how all jobs are affected, to a greater or lesser extent, by precariousness. This conceptualization also reveals marked social patterns in which women, young people and immigrants are most affected by precarious employment [14,24,38]. The 2 most widely used models are EPRES [10] and the quality of work life model [24], both based on the same theoretical framework. Nonetheless they are used differently in practice; one seeks to provide a measure of precariousness [14], while the other assesses the dimensions separately and evaluates their interaction [38].

The characteristics of precarious employment and its association with workers' health are widely determined by the macro-structural context [5]. Despite this, only 2 studies out of 13 that compared several European Union member states stratified the analysis by welfare state using Korpi's typology based on family policies [56], and concluded that it could be a relevant factor in the association between precarious employment and health [28,38]. These results are in line with a recent review [20]. The association between precariousness and mental health may differ depending on the type of the welfare state, due to different levels of social protection [28]. Further, including the welfare state characteristics in such analyses may explain some of the differences between men and women in terms of vulnerability associated with precarious employment (and its impact on health) [15,38]. Epidemiological designs integrating contextual variables are needed to shed some light on the complex theoretical framework linking macro-level factors, precarious employment and health.

The current economic recession is not an isolated economic phenomenon but it involves long-term consequences for the labor market, employment and working conditions, as well as workers' health [57]. Thus, more comparative studies are also needed to include and further explore the impact of the economic crisis on the growth of precarious working conditions and its association with mental health, as only 5 of the studies, 2 of which were from Southern Europe, attempted to give an account of this. On the other hand, only 7 studies considered workers' social and family support, and/or household situation. 
Only 3 concluded that social and family support reduces the impact of precarious employment on individuals' mental health $[19,44,45]$, a finding that is consistent with the existing scientific literature [9,58]. Two studies [42,43] found an insignificant impact of social support on the association between precarious employment and workers' mental health. Thus, the research on the influence of social and family support, in the precarious employment association with workers' mental health, is still in its infancy. There remain numerous areas to explore further in this line of research, such as the impact of precarious employment according to household composition, whether there are dependants, and whether other members of the household are unemployed or in precarious employment.

The studies analyzed propose different causal mechanisms underlying the relationship between precarious employment and mental health. The most widely considered, and not usually accounted for in the precarious employment theory, is the breadwinner model. It explains the gender differences in mental health due to precarious employment in relation to the traditional roles of men and women regarding work and family: men's life is more centered around paid work because they earn the family wage, while women do domestic and care labor. Thus, men's mental health depends more on their situation on the labor market $[14,17,24,32,38,59,60]$. This could be especially relevant in the contexts where the traditional family model is still dominant $[15,28,38]$.

The other exposed pathways are in line with those exposed in the introduction and in previous reviews $[4,12]$. First, the manifest and latent functions deprivation model, specifically pointing out that precarious employment implies fewer financial rewards [59,61-63], and a weakening of the employment latent functions, such as social integration, social and work status and identity, and self-fulfillment [27,63-65]. Second, the higher exposition of precarious employees to harmful working conditions. For example, precarious workers, in order to keep their employment, may accept worse working conditions, as well as intensify their work pace and increase their working time $[24,33,43,66]$. Finally, the lack of control that precarious workers have over their work; the future uncertainty and inability to prepare plans, as well as to achieve certain goals, can have negative effects on workers' mental health [63,67].

Taking into account the gender-based segmentation on the labor market, and the glass ceiling and sticky floor which characterize it [68], the higher percentage of women with precarious employment [38], and the gender-based division of domestic-family work [69], precarious employment and its impact on mental health should be interpreted from a gender-sensitive perspective. Nevertheless, still only around half of the studies included in this review contained sex-stratified analyses. Despite a growing tendency to include this perspective, in a review by Quinlan et al. [12] on the same topic, sex stratification was only performed in 9 out of 93 studies. Furthermore, studies draw contradictory conclusions regarding sex-dependent differences in the impact of precarious employment on health, some suggesting that precarious employment is more damaging for women $[15,24]$ while others that it is more damaging for men [30-33,35]. It is still a neglected and not well-understood topic.

Finally, there are very few studies proposing and discussing (both theoretically and empirically) a multidimensional definition, and conceptual frameworks that specify the micro- and macro-level pathways linking precarious employment and mental health. Thus, the political proposals described in the studies mainly consist of improving employment and working conditions or extending severance packages and unemployment benefits. There seems to be a need for deeper reflection to understand precarious employment as a well-defined multidimensional concept, and as a potentially modifiable risk factor, to enable the design of public policies to minimize the extent of employment precariousness, and the development of measures to reduce its impact on health. 


\section{Limitations and strengths}

This review has some limitations. Notably, the large number of cross-sectional studies included implies that the results must be interpreted with caution since causality cannot be established with certainty, and the influence of the healthy worker effect cannot be ruled out. Although precarious workers are difficult to follow longitudinally, it seems necessary to analyze individuals' employment history and investigate how changes from one working situation to another affect their mental health, and whether such effects are reversible (as found in some longitudinal studies on organizational restructuring processes). Extending the analysis to include changes over time is a challenge, but at the same time necessary to properly assess the impact of precarious employment on workers' mental health.

Further, a meta-analysis was not conducted. Although this type of analysis could have provided a clearer picture, it was not feasible due to differences between the studies when it comes to definitions of precarious employment, dimensions of mental health, study designs and statistical procedures. Another limitation, and potentially a source of bias, was the use of the same cohort or database in several of the studies included. Finally, the review focuses on peerreviewed studies. Thus, although this type of literature is relevant and informative, reports and articles published in non-peer-reviewed journals and other grey literature were not included. Lastly, publication bias may influence the interpretation of the findings, so caution is necessary. However, as the results of the included studies analyzing similar exposures were not homogeneous, the risk of major publication bias may be considered moderate.

The main strength of this research was the fact that it provides a systematic review of an issue of great political, economic and social importance, namely the association between precariousness (considering its different dimensions) and workers' mental health. Further, it is a review of the studies published in the last 8 years, and most of the studies included analyzed data collected since the crisis of 2008.
Precariousness has become the normative model of employment, with repercussions on both the health and social life of workers. There continues to be a risk that the numerous definitions and theoretical approaches used mask the scale of the problem and weaken the capacity for political action concerning preventive measures. A standardized multidimensional definition of precarious employment is crucial. Given the above this review sought to clarify the main research results in the scientific literature, in order to set out the key definitions, findings and their limitations.

\section{CONCLUSIONS}

The scientific articles included in this review demonstrate that precariousness, as reflected both in the analysis of its main dimensions separately and in multidimensional approaches, is associated with mental health problems. Most of the studies analyzing job insecurity, temporariness and multidimensional approaches reported a significant association with mental health. Nevertheless, results for working time arrangements and downsizing are inconclusive. Sex stratification was only performed in 9 out of 93 studies. Furthermore, studies draw contradictory conclusions regarding sex-dependent differences in the impact of precarious employment on health.

Theoretical frameworks integrating both contextual and individual variables are needed to shed some light on the complex theoretical framework linking macro-level factors, precarious employment and health.

There seems to be a need for deeper reflection to understand precarious employment as a well-defined multidimensional concept, and as a potentially modifiable risk factor, to enable the design of public policies to minimize the extent of employment precariousness and the development of measures to reduce its impact on health.

\section{ACKNOWLEDGMENTS}

The authors specially thank Amaia Bacigalupe for revision of the manuscript and for recommendations to improve it. 


\section{REFERENCES}

1. Comision on Social Determinants of Health. Closing the gap in a generation: Health equity through action on the social determinants of health. Geneva: World Health Organization; 2008.

2. Ferrie J, Westerlund H, Virtanen M, Vahtera J, Kiwimaki M. Flexible labor markets and employee health. Scand J Work Environ Health. 2008;Suppl 6:98-110.

3. Miguélez F, Prieto C. [Transformaciones del empleo, flexibilidad y relaciones laborales en Europa]. Política Soc. 2009;46(1-2):275-87. Spanish.

4. Benach J, Vives A, Amable M, Vanroelen C, Tarafa G, Muntaner C. Precarious employment: understanding an emerging social determinant of health. Annu Rev Public Health. 2014;35:229-53, https://doi.org/10.1146/annurev-publhealth032013-182500.

5. Kim T, von dem Knesebeck O. Perceived job insecurity, unemployment and depressive symptoms: a systematic review and meta-analysis of prospective observational studies. Int Arch Occup Environ Health. 2016;89(4):561-73, https://doi. org/10.1186/s12889-015-2313-1.

6. Tompa E, Scott-Marshall H, Dolinschi R, Trevithick S, Bhattacharyya $\mathrm{S}$. Precarious employment experiences and their health consequences: towards a theoretical framework. Work. 2007;28(3):209-24.

7. Amable M, Benach J, González S. [La precariedad laboral y su repercusión sobre la salud: conceptos y resultados preliminares de un estudio multimétodos.] Arch Prev Riesgos Labor. 2001;4(4):169-84. Spanish.

8. Rodgers G, Rodgers J. Precarious jobs in labour market regulation : the growth of atypical employment in Western Europe. Geneva: International Institute for Labour Studies; 1989.

9. Lewchuk W, Clarke M, de Wolff A. Working without commitments: precarious employment and health. Work Employ Soc. 2008;22(3):387-406, https://doi.org/10.1177/09500 17008093477.

10. Vives A, Amable M, Ferrer M, Moncada S, Llorens C, Muntaner C, et al. The Employment Precariousness Scale
(EPRES): psychometric properties of a new tool for epidemiological studies among waged and salaried workers. Occup Environ Med. 2010;67(8):548-55, https://doi.org/10. 1136/oem.2009.048967.

11. Virtanen M, Kivimäki M, Joensuu M, Virtanen P, Elovainio M, Vahtera J. Temporary employment and health: a review. Int J Epidemiol. 2005;34(3):610-22, https://doi.org/ 10.1093/ije/dyi024.

12. Quinlan M, Mayhew C, Bohle P. The global expansion of precarious employment, work disorganization, and consequences for occupational health: a review of recent research. Int J Health Serv 2001;31(2):335-414, https://doi. org/10.2190/607H-TTV0-QCN6-YLT4.

13. Benach J, Vives A, Tarafa G, Delclos C, Muntaner C. What should we know about precarious employment and health in 2025 ? framing the agenda for the next decade of research. Int J Epidemiol. 2016;45(1):232-8, https://doi.org/10.1093/ije/dyv342.

14. Vives A, Amable M, Ferrer M, Moncada S, Llorens C, Muntaner C, et al. Employment precariousness and poor mental health: Evidence from Spain on a new social determinant of health. J Environ Public Health. 2013;2013:978656, https:// doi.org/10.1155/2013/978656.

15. De Moortel D, Vandenheede H, Muntaner C, Vanroelen C. Structural and intermediary determinants of social inequalities in the mental well-being of European workers: a relational approach. BMC Public Health. 2014;14(1):938, https://doi. org/10.1186/1471-2458-14-938.

16. Vancea M, Utzet M. How unemployment and precarious employment affect the health of young people: A scoping study on social determinants. Scand J Public Health. 2017;45(1): 73-84, https://doi.org/10.1177/1403494816679555.

17. Muntaner C, Solar O, Vanroelen C, Martínez JM, Vergara M, Santana V, et al. Unemployment, Informal Work, Precarious Employment, Child Labor, Slavery, and Health Inequalities: Pathways and Mechanisms. Int J Heal Serv. 2010;40(2):281-95, https://doi.org/10.2190/HS.40.2.h.

18. Messing K, Punnett L, Bond M, Alexanderson K, Pyle J, Zahm S, et al. Be the fairest of them all: challenges and 
recommendations for the treatment of gender in occupational health research. Am J Ind Med. 2003;43(6):618-29, https://doi.org/10.1002/ajim.10225.

19. Van Aerden K, Gadeyne S, Vanroelen C. Is any job better than no job at all? Studying the relations between employment types, unemployment and subjective health in Belgium. Arch Public Health. 2017;75:55, https://doi.org/10.1186/s13 690-017-0225-5.

20. Kim I-H, Muntaner C, Shahidi FV, Vives A, Vanroelen C, Benach J, et al. Welfare states, flexible employment, and health: A critical review. Health Policy (New York). 2012;104(2):99127, https://doi.org/10.1016/j.healthpol.2011.11.002.

21. Eurostat [Internet]. Luxembourg: Eurostat; 2016 [cited 2018 Sep 3]. Unemployment statistics - Statistics Explained. Available from: https://ec.europa.eu/eurostat/statistics-explained/index.php/Unemployment_statistics.

22. StucklerD, Reeves A, Loopstra R, Karanikolos M, McKee M. Austerity and health: the impact in the UK and Europe. Eur J Public Health. 2017;27(suppl 4):18-21, https://doi.org/ 10.1093/eurpub/ckx167

23. Tacconelli E. Systematic reviews: CRD's guidance for undertaking reviews in health care. Lancet Infect Dis. 2010;10(4): 226, https://doi.org/10.1016/S1473-3099(10)70065-7

24. Van Aerden K, Puig-Barrachina V, Bosmans K, Vanroelen C. How does employment quality relate to health and job satisfaction in Europe? A typological approach. SocSci Med. 2016;158: 132-40, https://doi.org/10.1016/j.socscimed.2016.04.017

25. National Heart, Lung and Blood Institute [Internet]. Bethesda: The Institute; 2016 [cited 2018 Aug 20]. Quality Assessment Tool for Observational Cohort and Cross-Sectional Studies. Available from: https://www.nih.gov/about-nih/whatwe-do/nih-almanac/national-heart-lung-blood-institute-nhlbi.

26. Brenner MH, Andreeva E, Theorell T, Goldberg M, Westerlund $\mathrm{H}$, Leineweber $\mathrm{C}$, et al. Organizational Downsizing and Depressive Symptoms in the European Recession: The Experience of Workers in France, Hungary, Sweden and the United Kingdom. PLoS One. 2014;9(5):19, https://doi. org/10.1371/journal.pone.0097063.
27. Andreeva E, Brenner MH, Theorell T, Goldberg M. Risk of psychological ill health and methods of organisational downsizing: a cross-sectional survey in four European countries. BMC Public Health. 2017;7(1):758, https://doi.org/10.1186/ s12889-017-4789-3.

28. Artazcoz L, Cortes I, Benavides FG, Escriba-Aguir V, Bartoll X, Vargas H, et al. Long working hours and health in Europe: Gender and welfare state differences in a context of economic crisis. Health Place. 2016;40:161-8, https://doi. org/10.1016/j.healthplace.2016.06.004.

29. De MoortelD, Thévenon O, De Witte H, Vanroelen C. Working Hours Mismatch, Macroeconomic Changes, and Mental Wellbeing in Europe. J Health Soc Behav [Internet]. 2017;58(2): 217-31, https://doi.org/10.1177/0022146517706532.

30. Wahrendorf M, Blane D, Bartley M, Dragano N, Siegrist J. Working conditions in mid-life and mental health in older ages. Adv Life Course Res. 2013;18(1):16-25, https:// doi.org/10.1016/j.alcr.2012.10.004.

31. Sousa E, Agudelo-Suárez A, Benavides FG, Schenker M, García AM, Benach J, et al. Immigration, work and health in Spain: the influence of legal status and employment contract on reported health indicators. Int J Public Health. 2010;55(5):443-51, https://doi.org/10.1007/s00038-010-0141-8.

32. Arias-de la Torre J, Artazcoz L, Molina AJ, Fernández-Villa T, Martín V. Inequalities in mental health in the working population of Spain: a National Health Survey-based study. Gac Sanit. 2016;30(5):339-44, https://doi.org/10.1016/j.gaceta. 2016.02.011.

33. Waenerlund A, Gustafsson P, Virtanen P, Hammarström A. Is the core-periphery labour market structure related to perceived health? findings of the Northern Swedish Cohort. BMC Public Health. 2011;11(1):956, https://doi.org/10.1186/14712458-11-956.

34. Schütte S, Chastang J-F, Parent-Thirion A, Vermeylen G, Niedhammer I. Psychosocial work exposures among European employees: explanations for occupational inequalities in mental health. J Public Health (Oxf). 2015;37(3):373-88, https://doi.org/10.1093/pubmed/fdv044. 
35. Niedhammer I, Lesuffleur T, Algava E, Chastang J-FF. Classic and emergent psychosocial work factors and mental health. Occup Med (Lond). 2015;65(2):126-34, https://doi. org/10.1093/occmed/kqu173.

36. Waenerlund A-K, Virtanen P, Hammarström A. Is temporary employment related to health status? Analysis of the Northern Swedish Cohort. Scand J Public Health. 2011;39(5):5339, https://doi.org/10.1177/1403494810395821.

37. Navarro A, Utzet M, Salas S, Llorens C, Molinero-Ruiz E, Moncada S. Specific psychosocial exposures for workers' mental health: A population-based study. Am J Ind Med. 2017;60(8):747-52, https://doi.org/10.1002/ajim.22733.

38. De Moortel D, Vandenheede H, Vanroelen C. Contemporary employment arrangements and mental well-being in men and women across Europe: a cross-sectional study. Int J Equity Healh. 2014;13(1):90, https://doi.org/10.1186/s12939-014-0090-6.

39. Niedhammer I, Sultan-Taïeb H, Chastang J, Vermeylen G, Parent-Thirion A. Fractions of cardiovascular diseases and mental disorders attributable to psychosocial work factors in 31 countries in Europe. Int Arch Occup Environ Health. 2014;87(4): 403-11, https://doi.org/10.1007/s00420-013-0879-4.

40. Cottini E, Ghinetti P. Employment insecurity and employees' health in Denmark. Heal Econ (United Kingdom). 2018;27(2):426-39, https://doi.org/10.1002/hec.3580

41. Murcia M, Chastang J-F, Niedhammer I. Psychosocial work factors, major depressive and generalised anxiety disorders: Results from the French national SIP study. J Affect Disord. 2013;146(3):319-27, https://doi.org/10.1016/j. jad.2012.09.014.

42. Virga D, Iliescu D. The well-being of Romanian workers in Spain: antecedents and moderators. Eur J Work Organ Psychol. 2017;26(1):149-59, https://doi.org/10.1080/1359432X. 2016.1225728.

43. Canivet C, Bodin T, Emmelin M, Toivanen S, Moghaddassi M, Östergren P-O, et al. Precarious employment is a risk factor for poor mental health in young individuals in Sweden: a cohort study with multiple follow-ups. BMC Public Health. 2016;16(1):687, https://doi.org/10.1186/s12889-016-3358-5.
44. Teixeira AF, Dias SF. Labor market integration, immigration experience, and psychologicaldistress in a multi-ethnic sample of immigrants residing in Portugal. Ethn Health. 2018;23(1): 81-96, https://doi.org/10.1080/13557858.2016.1246421.

45. Snorradóttir Á, Vilhjálmsson R, Rafnsdóttir GL, Tómasson K. Financial crisis and collapsed banks: psychological distress and work related factors among surviving employees - a nation-wide study. Am J Ind Med. 2013;56(9):1095106, https://doi.org/10.1002/ajim.22210.

46. Benach J, Julià M, Tarafa G, Mir J, Molinero E, Vives A. Multidimensional measurement of precarious employment: social distribution and its association with health in Catalonia (Spain). Gac Sanit. 2015;29(5):375-8, https://doi. org/10.1016/j.gaceta.2015.04.002.

47. Sverke M, Hellgren J, Näswall K. No security: a meta-analysis and review of job insecurity and its consequences. J Occup Health Psychol. 2002;7(3):242-64.

48. Goudswaard A, Andries F. Employment status and working conditions. Luxembourg: Office for Official Publications of the European Communities; 2002.

49. Hünefeld L, Köper B. Fixed-term Employment and Job Insecurity (JI) as Risk factors for Mental Health. A Review of International Study Results. E-Journal Int Comp Labour Stud. 2016;5(3):28.

50. Falkenberg H, Fransson EI, Westerlund H, Head JA. Shortand long-term effects of major organisational change on minor psychiatric disorder and self-rated health: results from the Whitehall II study. Occup Environ Med. 2013;70(10):68896, https://doi.org/10.1136/oemed-2013-101385.

51. Osthus S. Health effects of downsizing survival and job loss in Norway. Soc Sci Med. 2012 Sep;75(5):946-53, https:/doi. org/10.1016/j.socscimed.2012.04.036.

52. Virtanen M, Jokela M, Madsen IEH, Hanson LLM, Lallukka T, Nyberg ST, et al. Long working hours and depressive symptoms: systematic review andmeta-analysis of published studies and unpublished individual participant data. Scand J Work Environ Heal. 2018;44(3):239-50, https://doi. org/10.5271/sjweh.3712. 
53. Mauss D, Litaker D, Jarczok MN, Li J, Bosch JA, Fischer JE. Anti-clockwise rotating shift work and health: would you prefer 3-shift or 4-shift operation? Am J Ind Med. 2013;56(5):599-608, https://doi.org/10.1002/ajim.22157.

54. Schütte S, Chastang J-F, Malard L, Parent-Thirion A, Vermeylen G, Niedhammer I. Psychosocial working conditions and psychological well-being among employees in 34 European countries. Int Arch Occup Environ Health. 2014;87(8):897907, https://doi.org/10.1007/s00420-014-0930-0.

55. Siegrist J, Marmot M. Health inequalities and the psychosocial environment-two scientific challenges. Soc Sci Med. 2004;58(8):1463-73, https://doi.org/10.1016/S0277-9536(03) 00349-6.

56. Korpi W. Faces of Inequality: Gender, Class, and Patterns of Inequalities in Different Types of Welfare States. Soc Polit Int Stud Gender State Soc. 2000;7(2):127-91, https://doi. org/10.1093/sp/7.2.127.

57. Barlow P, Reeves A, McKee M, Stuckler D. Austerity, precariousness, and the health status of Greek labour market participants: Retrospective cohort analysis of employed and unemployed persons in 2008-2009 and 2010-2011. J Public Health Policy. 2015;36(4):452-68, https://doi.org/10.1057/jphp.2015.25.

58. Vosko LF. Precarious Employment: Towards an Improved Understanding of Labour Market Insecurity. In: Vosko LF, editor. Precarious Employment. Understanding Labour Market Insecurity in Canada. Montreal: McGill-Queen's Press; 2006.

59. Fiori F, Rinesi F, Spizzichino D, Di Giorgio G. Employment insecurity and mental health during the economic recession: An analysis of the young adult labour force in Italy. Soc Sci Med. 2016;153:90-8, https://doi.org/10.1016/j.socscimed.2016.02.010.

60. Afonso P, Fonseca M, Pires JF. Impact of working hours on sleep and mental health. Occup Med (Lond). 2017;67(5): 377-82, https://doi.org/10.1093/occmed/kqx054.

61. Blanquet M, Labbe-Lobertreau E, Sass C, Berger D, Gerbaud L. Occupational status as a determinant of mental health inequities in French young people: is fairness needed? Results of a cross-sectional multicentre observational survey. Int J Equity Health. 2017;16(1):142, https://doi.org/ 10.1186/s12939-017-0634-7.

62. Canivet C, Aronsson G, Bernhard-Oettel C, Leineweber C, Moghaddassi M, Stengard J, et al. The negative effects on mental health of being in a non-desired occupation in an increasingly precarious labour market. SSM - Popul Heal. 2017;3:516-24, https://doi.org/10.1016/j.ssmph.2017.05.009.

63. Robert G, Martinez J, Garcia AMA, Benavides FFGF, Ronda E, Miguel Martinez J, et al. From the boom to the crisis: changes in employment conditions of immigrants in Spain and their effects on mental health. Eur J Public Health. 2014;24(3):404-9, https://doi.org/10.1093/eurpub/cku020.

64. Griep Y, Kinnunen U, Natti J, Nele DC, Mauno S, Makikangas A, et al. The effects of unemployment and perceived job insecurity: a comparison of their association with psychological and somatic complaints, self-rated health and life satisfaction. Int Arch Occup Environ Health. 2016;89(1): 147-62, https://doi.org/10.1007/s00420-015-1059-5.

65. Pirani E. On the Relationship Between Atypical Work(s) and Mental Health: New Insights from the Italian Case. Soc Indic Res. 2017;130(1, SI):233-52.

66. Sidorchuk A, Engstrom K, Johnson CM, Leeoza NK, Moller J. Employment status and psychological distress in a population-based cross-sectional study in Sweden: the impact of migration. BMJ Open. 2017;7(4):e014698, https:// doi.org/10.1136/bmjopen-2016-014698.

67. Julia M, Vives A, Tarafa G, Benach J. Changing the way we understand precarious employment and health: Precarisation affects the entire salaried population. Saf Sci. 2017;100(A, SI):66-73, https://doi.org/10.1016/j.ssci.2017.01.015.

68. Torns T, Recio C. Las desigualdades de género en el mercado de trabajo: entre la continuidad y la transformación. Rev Econ Crítica. 2012;14:178-202.

69. Moreno N, Moncada S, Llorens C, Carrasquer P. Double presence, paid work, and domestic-family work. New Solut. 2010;20(4):511-26, https://doi.org/10.2190/NS.20.4.h.

70. Reichert AR, Tauchmann H. Workforce reduction, subjective job insecurity, and mental health. J Econ Behav 
Organ.2017;133:187-212,https://doi.org/10.1016/j.jebo.2016. 10.018 .

71. Flint E, Cummins S, Wills J. Investigating the effect of the London living wage on the psychological wellbeing of low-wage service sector employees: a feasibility study. J Public Health (Oxf). 2014;36(2):187-93, https://doi.org/10.1093/pubmed/fdt093.

72. Boschman JS, van der Molen HF, Sluiter JK, Frings-Dresen MHW. Psychosocial work environment and mental health among construction workers. Appl Ergon. 2013;44(5):74855, https://doi.org/10.1016/j.apergo.2013.01.004.

73. Zoghbi-Manrique-de-Lara P, Verano-Tacoronte D, GuerraBaez RM. Well-being and behavior of hotel employees in the context of the currenteconomic crisis. Investig Tur. 2016;(11):96-115.

74. Magnusson Hanson LL, Chungkham HS, Ferrie J, Sverke M. Threats of dismissal and symptoms of major depression: a study using repeat measures in the Swedish working population. J Epidemiol Community Health. 2015;69(10):963-9, https://doi.org/10.1136/jech-2014-205405.

75. Meltzer H, Bebbington P, Brugha T, Jenkins R, McManus S, Stansfeld S. Job insecurity, socio-economic circumstances and depression. Psychol Med. 2010;40(8):1401-7, https://doi. org/10.1017/S0033291709991802.

76. Ten Have M, van Dorsselaer S, de Graaf R. The association between type and number of adverse working conditions and mental health during a time of economic crisis (2010-2012). Soc Psychiatry Psychiatr Epidemiol. 2015;50(6):899-907, https://doi.org/10.1007/s00127-015-1009-2.

77. Rajani NB, Giannakopoulos G, Filippidis FT. Job insecurity, financial difficulties and mental health in Europe. Occup Med. 2016;66(8):681-3, https://doi.org/10.1093/occmed/kqw111.

78. Buffel V, Dereuddre R, Bracke P. Medicalization of the Uncertainty? An Empirical Study of the Relationships between Unemployment or Job Insecurity, Professional Care
Seeking, and the Consumption of Antidepressants. Eur Sociol Rev. 2015;31(4):446-59, https://doi.org/10.1093/esr/ jcv004.

79. Virtanen P, Janlert U, Hammarström A. Exposure to temporary employment and job insecurity: a longitudinal study of the health effects. Occup Environ Med. 2011;68(8):570-4.

80. Cortes-Franch I, Escriba-Aguir V, Benach J, Artazcoz L. Employment stability and mental health in Spain: towards understanding the influence of gender and partner/marital status. BMC Public Health. 2018;18(1):425, https://doi. org/10.1186/s12889-018-5282-3.

81. Houdmont J, Randall R. Working hours and common mental disorders in English police officers. Occup Med (Lond). 2016;66(9):713-8, https://doi.org/10.1093/occmed/kqw166.

82. Vives A, Vanroelen C, Amable M, Ferrer M, Moncada S, Llorens $\mathrm{C}$, et al. Employment precariousness in Spain: prevalence, social distribution, and population-attributable risk percent of poor mental health. Int J Health Serv. 2011;41(4):625-46, https://doi.org/10.2190/HS.41.4.b.

83. Henseke G. Good jobs, good pay, better health? The effects of job quality on health among older European workers. Eur J Heal Econ. 2018;19(1):59-73, https://doi.org/10.1007/ s10198-017-0867-9.

84. Niedhammer I, Malard LL, Chastang J-F. Occupational factors and subsequent major depressive and generalized anxiety disorders in the prospective French national SIP study. BMC Public Health. 2015;15(1):200, https://doi.org/10.1186/ s12889-015-1559-y.

85. Kirves K, De Cuyper N, Kinnunen U, Nätti J. Perceived job insecurity and perceived employability in relation to temporary and permanent workers' psychological symptoms: A two samples study. Int Arch Occup Environ Health. 2011;84(8): 899-909, https://doi.org/10.1007/s00420-011-0630-y.

This work is available in Open Access model and licensed under a Creative Commons Attribution-NonCommercial 3.0 Poland License - http://creativecommons.org/ licenses/by-nc/3.0/pl/deed.en. 